\title{
Saraswati: An Extremely Massive 200 Megaparsec Scale Supercluster
}

\author{
Joydeep Bagchi ${ }^{1}$, Shishir Sankhyayan ${ }^{2}$, Prakash Sarkar ${ }^{3}$, Somak Raychaudhury ${ }^{1}$, Joe Jacob ${ }^{4}$, and Pratik Dabhade ${ }^{1}$ \\ ${ }^{1}$ The Inter-University Centre for Astronomy and Astrophysics (IUCAA), S.P. Pune University Campus, Post Bag 4, Pune 411007, India; joydeep@iucaa.in \\ ${ }^{2}$ Indian Institute of Science Education and Research (IISER), Dr. Homi Bhabha Road, Pashan, Pune 411008, India \\ ${ }^{3}$ Dept. of Physics, National Institute of Technology (NIT), NIT Campus, P.O. RIT, Jamshedpur 831014, India \\ ${ }^{4}$ Department of Physics, Newman College, Thodupuzha 685585, India \\ Received 2017 January 18; revised 2017 May 31; accepted 2017 June 9; published 2017 July 19
}

\begin{abstract}
Here we report the discovery of an extremely massive and large supercluster (called Saraswati ${ }^{5}$ ) found in the Stripe 82 region of SDSS. This supercluster is a major concentration of galaxies and galaxy clusters, forming a wall-like structure spanning at least $200 \mathrm{Mpc}$ across at redshift $z \approx 0.3$. This enormous structure is surrounded by a network of galaxy filaments, clusters, and large, $\sim 40-170 \mathrm{Mpc}$ diameter, voids. The mean density contrast $\delta$ (relative to the background matter density of the universe) of Saraswati is $\gtrsim 1.62$ and the main body of the supercluster comprises at least 43 massive galaxy clusters (mean $z=0.28$ ) with a total mass of $\sim 2 \times 10^{16} M_{\odot}$. The spherical collapse model suggests that the central region of radius $\sim 20 \mathrm{Mpc}$ and mass at least $4 \times 10^{15} M_{\odot}$ may be collapsing. This places it among the few largest and most massive superclusters known, comparable to the most massive "Shapley Concentration" $(z \approx 0.046)$ in the nearby universe. The Saraswati supercluster and its environs reveal that some extreme large-scale, prominent matter density enhancements formed $\sim 4 \mathrm{Gyr}$ in the past when dark energy had just started to dominate structure formation. This galactic concentration sheds light on the role of dark energy and cosmological initial conditions in supercluster formation, and tests the competing cosmological models.
\end{abstract}

Key words: cosmology: observations - galaxies: clusters: general - large-scale structure of universe

\section{Introduction}

In the leading paradigm of Cold Dark Matter $(\Lambda \mathrm{CDM})$ cosmology, large-scale structures assemble hierarchically through the gravitational clustering of matter. An interplay of dark matter and dark energy results in an intricate pattern of interconnected filaments, wall-like pancakes, and dense clusters surrounded by large near-empty void regions (Einasto 2014). Superclusters of galaxies are believed to be the largest concentrations of matter in the universe whose origin is still being debated (de Vaucouleurs 1956; Abell 1961; Jõeveer et al. 1978; Einasto et al. 1994). Occasionally spanning over a hundred megaparsecs (Mpc), superclusters usually display a pronounced filamentary or sheetlike morphology, and are surrounded by large almost empty regions called voids (Einasto 2014). Taken together, the supercluster-void distribution is commonly referred to as the "Cosmic Web" (Bond et al. 1996; Einasto 2014). One has been able to associate superclusters with a network of galaxies connected to a few rich cluster systems, as well as with denser and more massive structures characterized by the concentration of several very massive clusters. Contemporary catalogs of superclusters therefore range from relatively smaller systems such as the Hercules supercluster (Abell 1961) to the much more massive Shapley Concentration containing several major clusters and numerous groups (Raychaudhury 1989; Bardelli et al. 2000; Proust et al.

\footnotetext{
5 Saraswati (सरस्वती) is the ancient Indian goddess of knowledge, music, art, wisdom, and nature, muse of all creative endeavor. Historically, the Saraswati river is an important river goddess mentioned in the Rig'veda. The river could not be identified with any present day rivers. Saraswati played an important role in Indian culture, since Vedic Sanskrit and the first part of the Rig'veda are regarded to have originated when the Vedic people lived on its banks, during the second millennium BCE. The Sanskrit name also means "ever flowing stream with many pools." This may describe the present large-scale filamentary structure of galaxies located in the Zodiacal constellation of "Pisces," having many clusters and groups moving and merging together. (https://en.wikipedia. org/wiki/Saraswati.)
}

2006). More recently, a new supercluster called Laniakea, spanning $\sim 160 \mathrm{Mpc}$ across, is identified (Tully et al. 2014), which is home to our Milky Way galaxy. Another large, wall-like structure spanning $\sim 250 \mathrm{Mpc}$ was discovered in the SDSS-BOSS survey (the BOSS Great Wall) at redshift 0.47 (Lietzen et al. 2016).

Unlike clusters, superclusters have not yet virialized and so may retain some memory of their initial conditions that led to their formation. The assembly of the largest superclusters is still a matter of conjecture, with arguments being given both in favor of assembly through gravitational clustering (Park et al. 2012) and the more radical point of view that large superclusters should be viewed as rare objects (Sheth \& Diaferio 2011), which might even challenge the widely held Copernican principle (Liddle 2003). Therefore, large-scale matter overdensities represented by clusters and superclusters, especially those identified at early cosmological epochs $z \gtrsim 0.3$, (i) are sensitive probes of the presence of dark matter and dark energy at higher redshifts, (ii) shed light on how structure might form in the universe, and (iii) provide model-independent tests of general relativity on the largest scales by extending solar system tests by $8-10$ orders of magnitude (Jain \& Khoury 2010).

The primary focus of this paper is Saraswati, an unusually massive and large-scale $(\sim 200 \mathrm{Mpc})$ supercluster of galaxies at a mean redshift $z=0.28$, and its properties, identified in the Sloan Digital Sky Survey (SDSS-III) data. This wall-like supercluster is shown to be associated with at least 43 massive galaxy clusters and groups, of which a few extremely massive ones constitute its bound, central core region. Our observations show Saraswati to be highly unusual in terms of its morphology, mass, and richness, and in detection of localized non-thermal radio emission, relating to its complex dynamical state. We have highlighted the importance of our findings for ongoing cosmological studies of dark energy and the growth of large-scale structures in the universe. We have used the following cosmological parameters based on five-year WMAP 


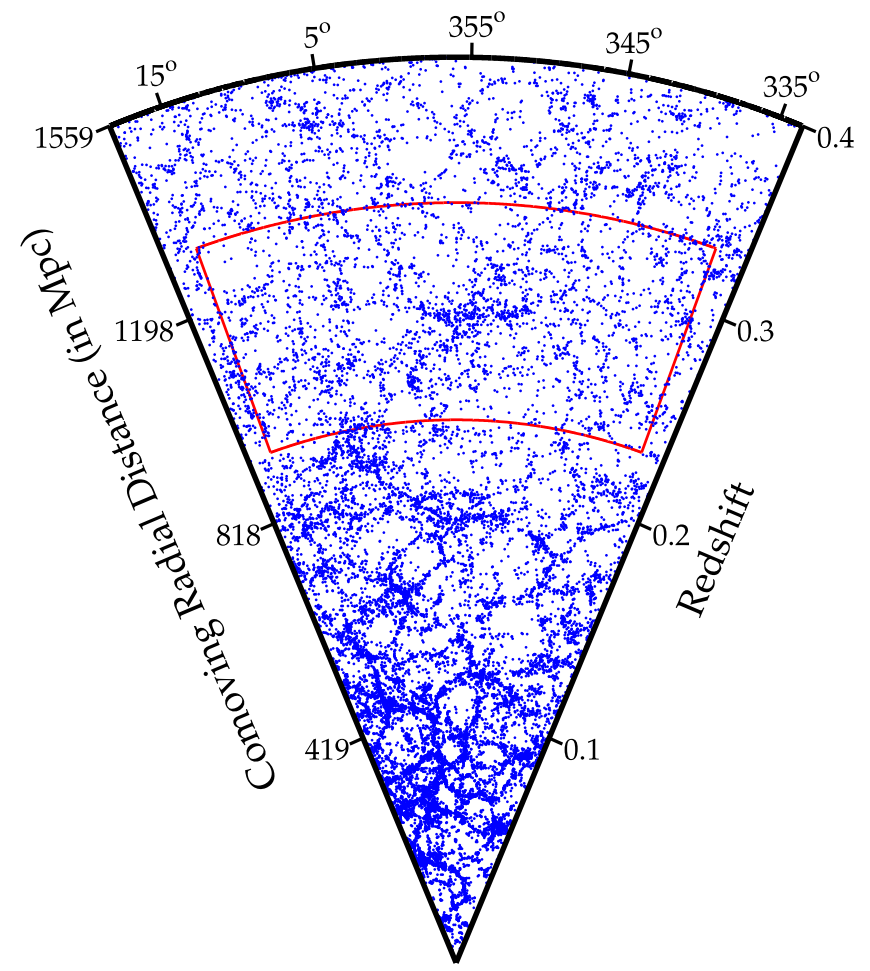

Figure 1. Redshift cone plot of SDSS galaxies (LBS sample; see text), extending from redshift $z=0$ to 0.4 , and R.A. width $45^{\circ}$, decl. width 2.5 , centered near Saraswati supercluster, the prominent large-scale structure within the red box. The small depth in decl. direction has been suppressed for clarity.

results (Komatsu et al. 2009); $\Omega_{M}=0.279, \Omega_{\Lambda}=0.721$, $\Omega_{R}=8.493 \times 10^{-5}, \omega_{0}=-1$, and $H_{0}=70.1 \mathrm{~km} \mathrm{~s}^{-1} \mathrm{Mpc}^{-1}$, which results in scale factor of $4.47 \mathrm{kpc} \operatorname{arcsec}^{-1}$ for a redshift of 0.3 . Below, $M_{n}$ and $R_{n}$ denote the total mass and radius, respectively, corresponding to a total density contrast $n \rho(z)$, where $\rho(z)$ is the critical density of the universe at redshift $z$.

\section{Identifying an Exceptionally Massive and Large-scale Supercluster}

Our primary results, pertaining to the discovery of a $\sim 200$ Mpc scale, massive supercluster (Saraswati) in the distribution of galaxies is shown in Figures 1, 4, 5, and the subsequent figures. A redshift cone plot of SDSS galaxies, between $z=0$ to 0.4 , showing the large supercluster of galaxies at $z \approx 0.3$ is shown in Figure 1.

Stripe 82. The galaxy redshift data used in this work is from the central part of the Stripe 82 region of the Sloan Digital Sky Survey (SDSS-III DR12) (Stoughton et al. 2002; Alam et al. 2015). The data was taken with the multi-fiber spectrograph mounted on the Sloan $2.5 \mathrm{~m}$ telescope at Apache Point Observatory, NM. Stripe 82 is currently the largest threedimensional spectroscopic sample of galaxies with a high sampling density, and photometrically up to two magnitudes deeper than the standard SDSS images. Stripe 82 covers a $\sim 270$ degree $^{2}$ area along the celestial equator in the Southern Galactic Cap and spans $310^{\circ}<$ R.A. $<59^{\circ}$ and $-1.25 \leqslant$ decl. $\leqslant 1.25$. A major supercluster was noticed while exploring the large-scale distribution of galaxies surrounding an unusual filamentary, merging galaxy cluster $\mathrm{ZwCl} 2341.1+0000(z=0.27)$. This massive cluster also shows large-scale diffuse synchrotron radio emission from structure formation shocks, and thus is a prominent target of several studies (Bagchi et al. 2002; van Weeren et al. 2009; Boschin et al. 2013).

The entire Stripe 82 is not uniformly sampled (spectroscopically), but contains data from several projects. We use two largest samples of spectroscopic galaxies: the LOWZ sample and the LBS (LEGACY-BOSS-SOUTHERN) sample described in more details below. We have $k$-corrected the absolute magnitudes of galaxies from both samples by using the K-CORRECT version 4.3 software (Blanton \& Roweis 2007). The $k$-corrected magnitudes are calculated for rest frame at $z=0.33$. We have retained only those galaxies in our analysis that have clean photometry, clean spectra, and redshift errors $<1 \%$. The spectra of a few galaxies from our samples are shown in Figure 15. The apparent magnitudes are SDSS $c$ Model magnitudes corrected for extinction. cModel magnitudes are composite model magnitudes, calculated from the best-fitting linear combination of a de Vaucouleurs and an exponential luminosity profile.

\subsection{LOWZ Spectroscopy Data from SDSS DR12}

In order to evaluate the properties of the new supercluster (at a mean $z=0.288$ ) and explore the cosmic web of galaxies, clusters, and voids surrounding it, we first extracted LOWZ data for galaxies from the Baryon Oscillation Spectroscopic Survey (BOSS; Ahn et al. 2012) of SDSS DR12, within a wedge of R.A. and decl. range $336^{\circ} \leqslant$ R.A. $\leqslant 16^{\circ},-1.25 \leqslant$ decl. $\leqslant 1.25$ (limits of survey) and spectroscopic redshift range $0 \leqslant z \leqslant 0.6$, within the Stripe 82 central region. BOSS targets LOWZ $(z \leqslant 0.6)$ galaxies using a set of colormagnitude cuts that follow the predicted evolution of a passively evolving stellar population with redshift. The selected galaxies are the brightest and reddest of the low-redshift galaxy population, and the targeting cuts are similar to those of SDSSI/II Cut-I Luminous Red Galaxies (LRGs). The LOWZ sample extends the SDSS-I/II LRGs by selecting fainter galaxies $(16<r<19.6)$, thereby increasing the number density. Due to a bug in the target selection, LOWZ galaxies were incorrectly targeted during the initial stages of the BOSS survey (affecting all data taken until June 2010). In order to form a uniformly targeted sample of LOWZ galaxies, we use only those galaxies whose TILEID $\geqslant 10324$. A volume-limited galaxy sample was constructed by restricting the cModel $r$-band absolute magnitude to $M_{r} \leqslant-21.53$ and the redshift to $z \leqslant 0.33$. From this volume-limited sample we make a subsample with $336^{\circ} \leqslant$ R.A. $\leqslant 16^{\circ}, \quad-1.25 \leqslant$ decl. $\leqslant$ +1.25 and $0.23 \leqslant z \leqslant 0.33$, which is centered on the Saraswati supercluster (hereafter called the "analysis wedge/ region"). This way we get a total of 625 spectroscopic galaxies in this subsample. For further analysis we converted the SDSS angular coordinates $(\alpha, \delta)$ and redshifts $(z)$ of galaxies to comoving cartesian coordinates $(X, Y, Z)$. The two-dimensional distribution of these galaxies in comoving coordinates is shown in Figure 2.

\subsection{BOSS-LEGACY-SOUTHERN Data}

The LOWZ data is uniformly sampled but it is fairly sparse. In order to obtain higher density of galaxies within our analysis wedge (as above), we combine all available spectroscopic galaxies with clean spectra and clean photometry taken from "LEGACY," "BOSS," and "SOUTHERN" programs of the SDSS-III DR12 database. Only these three programs were selected because the galaxies in these programs were deemed to 


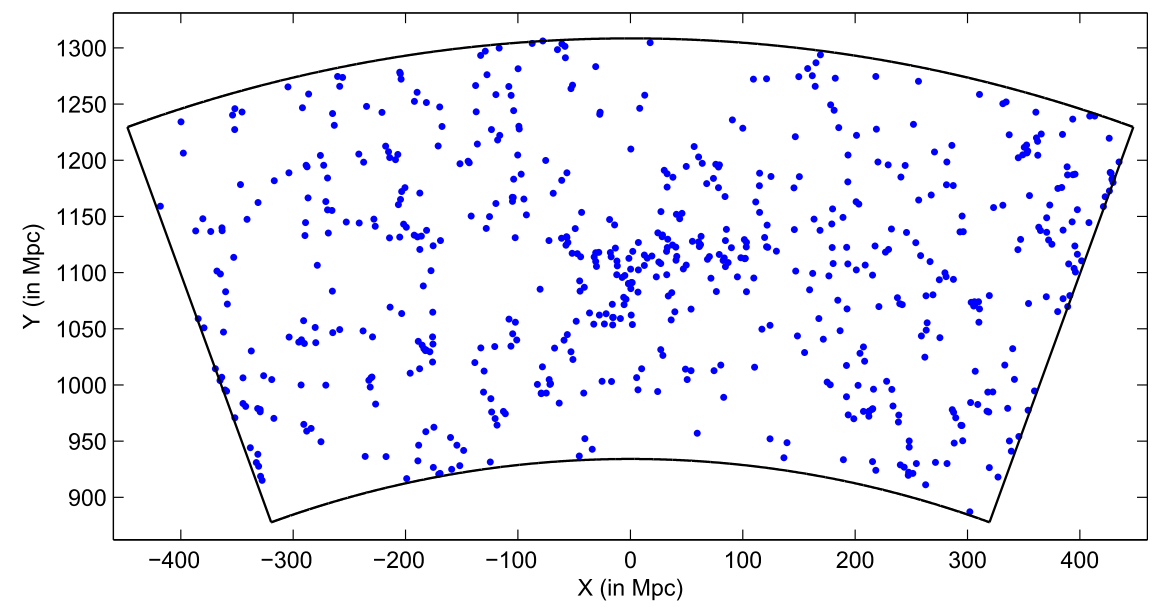

Figure 2. Distribution of 625 galaxies in the subsample of LOWZ volume-limited sample in comoving coordinates, projected on the two-dimensional $X-Y$ plane. The small depth in Z-direction (along decl.) has been suppressed.

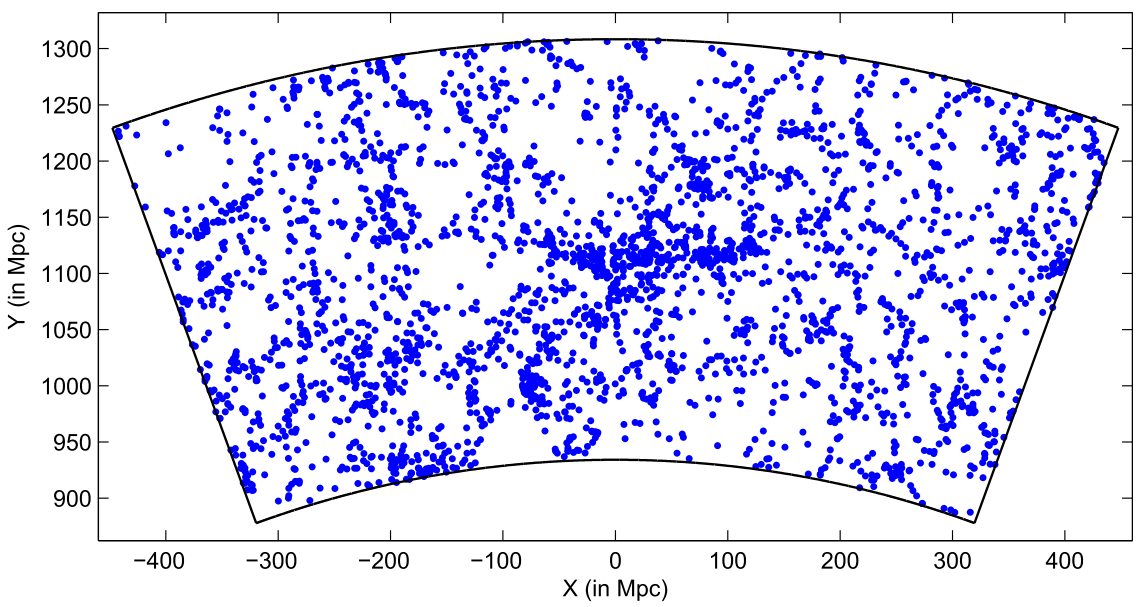

Figure 3. LEGACY-BOSS-SOUTHERN (LBS) sample of 3016 galaxies plotted in comoving coordinates, projected on the two-dimensional $X-Y$ plane. The small depth in the Z-direction (along decl.) has been suppressed.

be more or less uniformly distributed within our sample region. This gives a total of 3016 galaxies, approximately five times the size of the LOWZ sample. Hereafter, we shall refer to this larger sample of 3016 galaxies as the LBS sample. Figure 3 shows the distribution of galaxies from the LBS sample within our wedge in comoving coordinate space.

In both samples, a prominent, large-scale $(\sim 200 \mathrm{Mpc})$ density enhancement of galaxies at mean $z \sim 0.28, X \approx 0$ $\mathrm{Mpc}$, and $Y \approx 1100 \mathrm{Mpc}$ is clearly visible, which identifies the Saraswati supercluster, the focus of our present study.

\section{Method of Analysis}

Superclusters are the primary large-scale galactic structures made of the concentration of smaller galaxy groups and clusters. The identification of superclusters requires extensive spectroscopic redshift data taken over vast areas of the sky, targeting galaxies in both high- and low-density regions of the cosmic web. There are generally two widely used methods to identify the superclusters; first, identifying overdense structures based on the Friends-of-Friends (FoF) algorithm (Huchra \& Geller 1982; Martínez \& Saar 2002) with a particular linking length, which can be applied both to individual galaxies and to groups and clusters of galaxies, and second, using the smoothed density field approach (Einasto 2014).
We have adopted the FoF approach to identify overdense structures in the SDSS galaxy samples. In an FoF algorithm, a linking length $(l)$ is chosen for the galaxy distribution. If the separation between two galaxies is less than or equal to $l$, these two galaxies are considered linked and part of the same group (cluster), otherwise not. Defined in this manner the size of a supercluster naturally becomes sensitive to the linking length used to determine it. The $l$ for a given point distribution (galaxies) is generally chosen such that maximum number of clusters are obtained given a predetermined limit to the minimum number of galaxies required in a cluster.

\section{Results}

\subsection{Identifying Clusters and Superclusters in SDSS Samples \\ 4.1.1. Clustering Analysis-LOWZ Galaxies}

We will now perform the cluster analysis (finding clusters) on the subsample of 625 galaxies from the LOWZ data. To classify clusters, the FoF algorithm is applied. The maximum number of 46 clusters, with at least four galaxies in a cluster, is obtained at comoving linking length $l \approx 19 \mathrm{Mpc}$. Hence we used $19 \mathrm{Mpc}$ as the linking length for the cluster analysis. This reflects the sparse sampling of LOWZ data. The largest cluster found this way is the Saraswati supercluster with 100 galaxies in it. Figure 4 


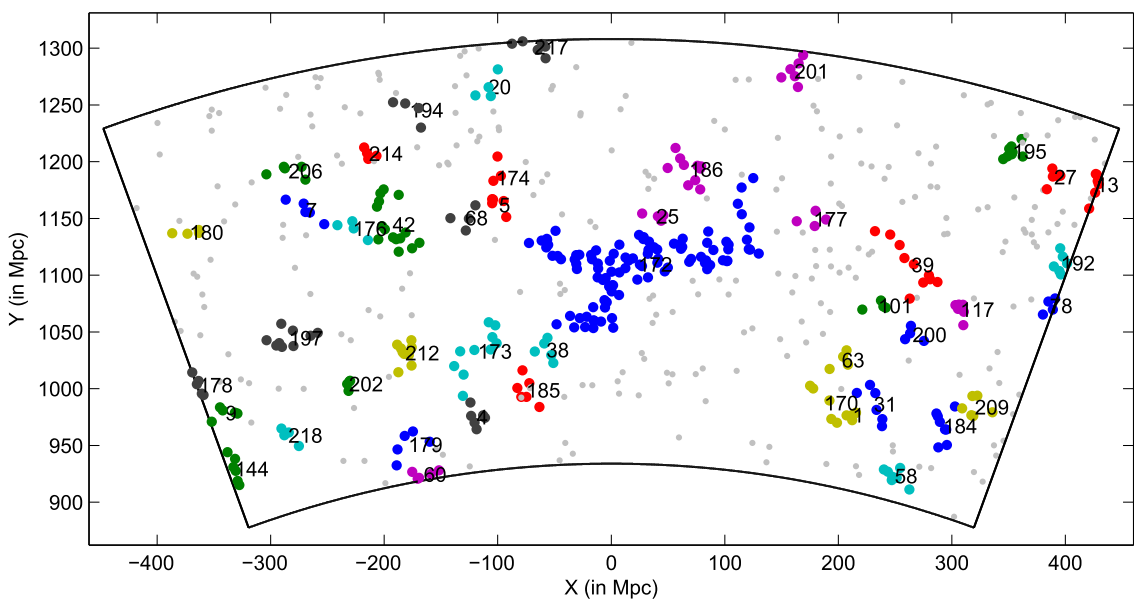

Figure 4. Clusters found in the LOWZ subsample by the FoF algorithm for comoving linking length of $l=19 \mathrm{Mpc}$. The minimum number of galaxies in a cluster is four. The clusters are shown in colors and marked with index numbers. In spite of sparse sampling the largest cluster identified is the Saraswati supercluster (index number 172) near the center shown in blue, containing $\approx 100$ galaxies. Gray dots are galaxies that are not part of any FoF cluster.

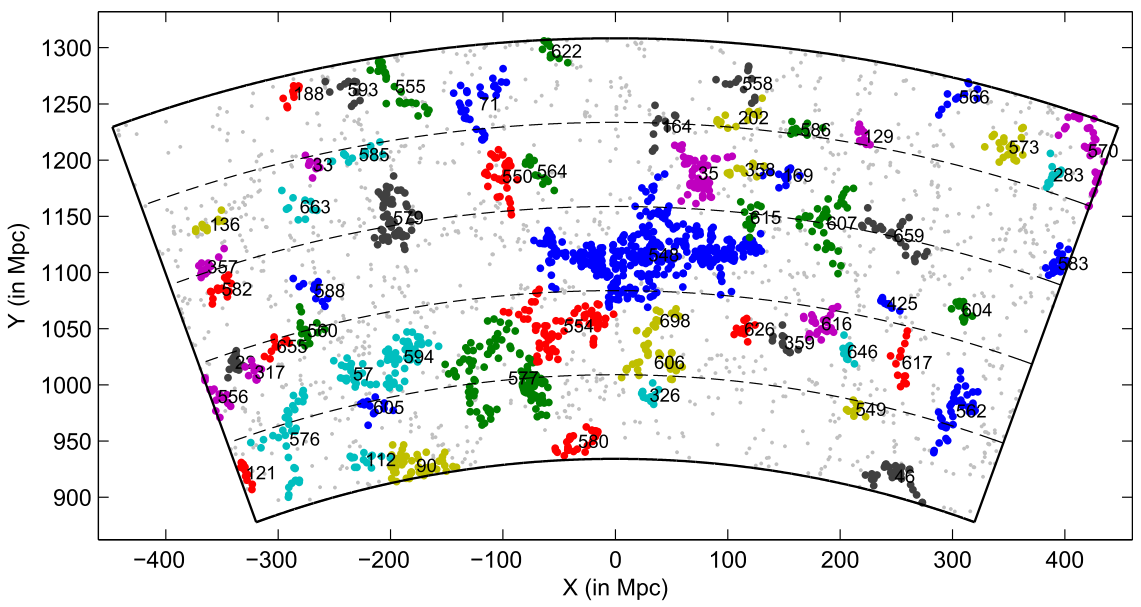

Figure 5. Clusters identified in the LBS sample using an FoF algorithm and a comoving linking length $l_{o}=12 \mathrm{Mpc}$. The minimum number of galaxies in each cluster is 10. Dotted arcs divide the wedge into five redshift bins as mentioned in Section 4.1.2. Different colors and indices indicate different clusters. The largest overdensity of galaxies found is Saraswati, spanning $\sim 200 \mathrm{Mpc}$ across (in blue color at the center) with $\approx 400$ galaxies in it and an index number 548 . Gray dots are galaxies that are not part of any FoF cluster.

shows all 46 FoF clusters, and the largest supercluster Saraswati is shown in blue at the center of the plot. Saraswati spans $\sim 200$ $\mathrm{Mpc}$ of comoving length across our line of sight.

\subsubsection{Clustering Analysis—LBS Galaxies}

Next we use the FoF algorithm to find clusters in the LBS sample of 3016 galaxies. Since this sample is not strictly uniformly sampled in redshift, we use local luminosity functions, i.e., the comoving number density per unit absolute magnitude, of the galaxies to give proper weights to galaxies so that unbiased clustering analysis can be performed using the $\mathrm{FoF}$ algorithm. The analysis region wedge is first divided into five constant comoving distance bins and the local luminosity functions of galaxies in each bin are calculated (see Figure 6). We then assign each galaxy (say $i$ th galaxy), having an absolute magnitude $M$, a weight $w_{i}$ according to the values of luminosity functions at $M$,

$$
w_{i}=\left[\frac{L F_{1, M}}{L F_{n, M}}\right]_{i}
$$

where $L F_{1, M}$ and $L F_{n, M}$ are the values of local luminosity functions of the lowest and the $n$th (the bin in which this $i$ th galaxy is located) comoving distance bins at magnitude $M$, respectively. Thus, the weight depends on both the absolute magnitude and the number density of the galaxy in the comoving distance bin. This will assign a weight $w=1$ to all galaxies in the lowest bin.

Next, we apply proper weights on the linking length between the two galaxies being linked. If $l_{o}$ is the selected linking length between galaxy $i$ and galaxy $j$ then the weighted linking length $l_{i j}$ will be

$$
l_{i j}=w_{i j} l_{o}
$$

where $w_{i j}$ is the weight between the $i$ th and $j$ th galaxies,

$$
w_{i j}=\frac{w_{i}^{\frac{1}{3}}+w_{j}^{\frac{1}{3}}}{2}
$$

where $w_{i}$ and $w_{j}$ are the weights associated with $i$ th and $j$ th galaxies according to the local luminosity functions (Equation (1) above).

Using this method, we get the largest number of clusters at $l_{o}=12 \mathrm{Mpc}$ with a minimum number of galaxies in a cluster to be 10 . Using these parameters, we identify a total of $60 \mathrm{FoF}$ galaxy clusters within the LBS analysis region. Once again, the largest cluster with a maximum number of linked galaxies, 


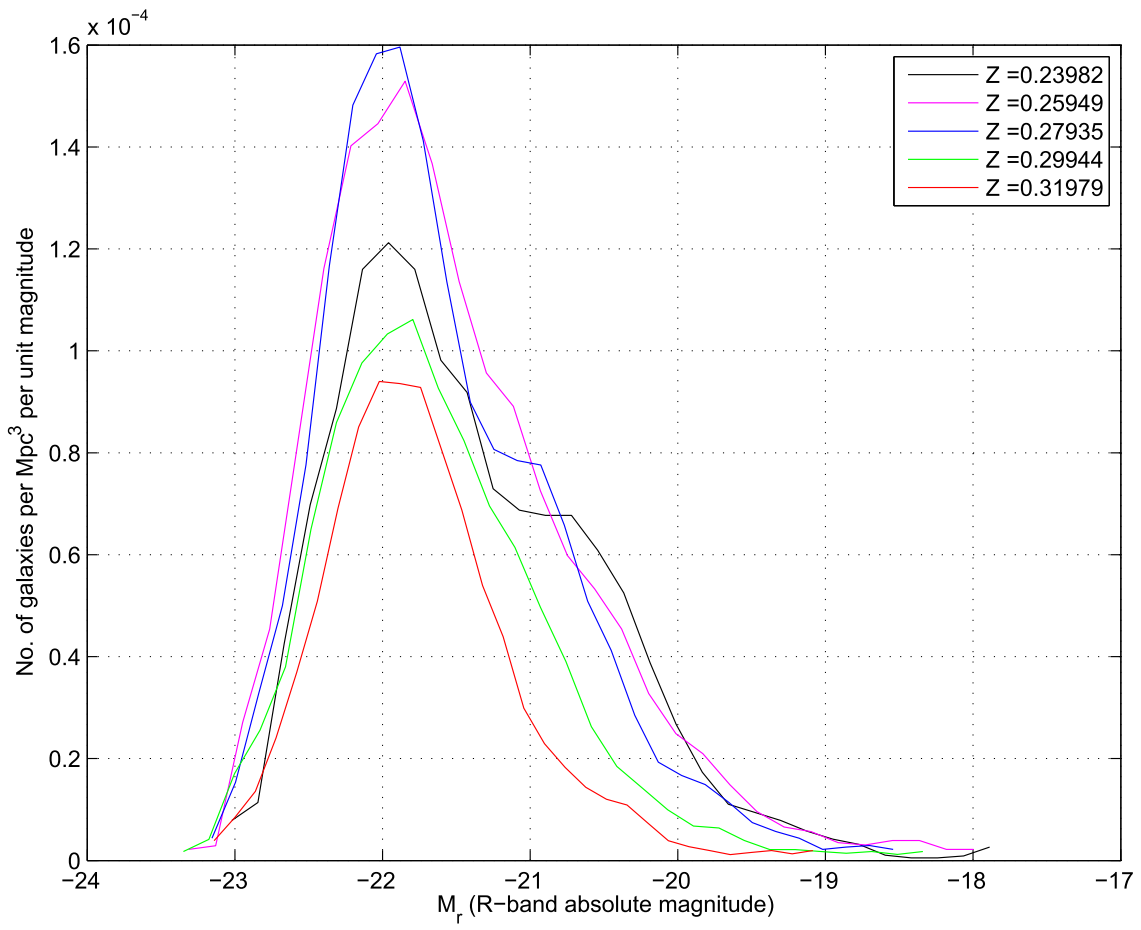

Figure 6. Local luminosity functions of LBS sample in five different redshift/comoving distance bins. Different color plots show the comoving number densities of galaxies per absolute cModel r-band magnitude. The mean redshift for each luminosity function plot is shown on top right corner.

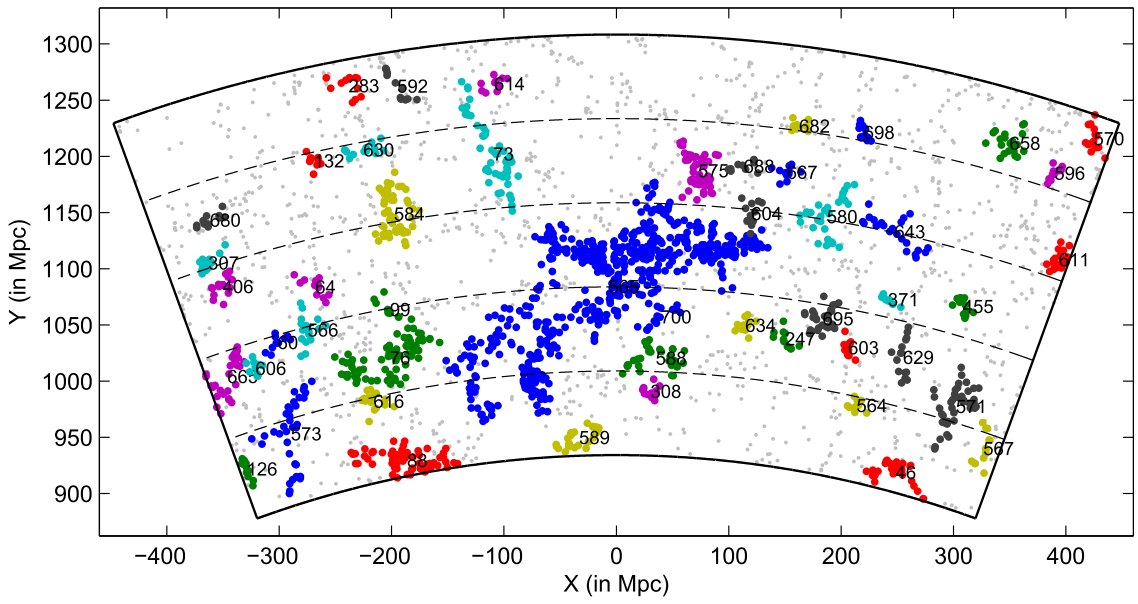

Figure 7. Clusters in LBS sample using FoF algorithm and a fixed (unweighted) linking length of $12 \mathrm{Mpc}$. The minimum number of galaxies in each cluster is 10 . Different colors and indices indicate different clusters. This analysis extends the Saraswati supercluster further toward lower redshifts by linking it with two nearby large galaxy filaments (in blue color at center).

naturally comes out to be the Saraswati supercluster with $\sim 400$ galaxies in it. In Figure 5, all our identified clusters are shown with various colors and using different index numbers. The Saraswati supercluster spanning $\approx 200 \mathrm{Mpc}$ across the line of sight $\left(\approx 9^{\circ}\right)$ stands out prominently in the middle of the plot. Moreover, even in limited sampling one gets a clear visual impression of the rich tapestry of filaments, galaxy clusters, and large voids of $\approx 40-170 \mathrm{Mpc}$ diameter surrounding the supercluster.

Figure 7 shows the FoF clusters using a fixed linking length of $12 \mathrm{Mpc}$ and with all weights set equal to 1 . The figure indicates that unweighted linking length further increases the extent of Saraswati downwards by linking it with two nearby large galaxy filaments. Whereas our clustering analysis with adaptive weighting scheme detects them as separate structures.

Therefore, based on this study, and with the firm identification of several massive galaxy clusters and voids near the
Saraswati supercluster in sections below, we have very little scope for doubt left that it is a real physical structure formed via cosmological processes, and not an artifact of chance alignment and projection effects.

\subsection{The Affect of Redshift Errors and Peculiar Velocities on Clustering Analysis}

As mentioned in Section 2, the spectroscopic redshift errors are less than $1 \%$ for all galaxies in our samples. The small redshift errors have a negligible affect on our clustering analysis. Another important factor is the Redshift Space Distortion (RSD), which is more significant for galaxies within the virial radius $r_{200}$ of a cluster. To take into account the affect of RSD, we calculated, for the LBS sample, using the virial masses $M_{200}$ and positions of WHL clusters (see Sections 5 
and 6.1), the RSD induced by these clusters in radial direction on the suurounding galaxies up to distances $r_{200}$. We corrected the comoving distances for all those galaxies which are within angular virial radius of the cluster and within a distance of $\left(v_{200} / H_{o}\right)$ from BCG (brightest cluster galaxy) in radial direction using the following equation,

$$
d_{\mathrm{gal}}=d_{\mathrm{BCG}}+\left(d_{\mathrm{gal}}^{*}-d_{\mathrm{BCG}}\right) \frac{r_{200}}{v_{200} / H_{o}}
$$

where $d_{\text {gal }}$ is the new comoving distance of galaxy, $d_{\text {gal }}^{*}$ is the old comoving distance of galaxy, $d_{\mathrm{BCG}}$ is the comoving distance of BCG, $r_{200}$ is the virial radius of the cluster, $v_{200}$ is the radial peculiar velocity component at $r_{200}$ due to mass $M_{200}$ $\left(v_{200}=\sqrt{G M_{200} / 3 r_{200}}\right)$, and $H_{o}$ is the Hubble parameter at $z=0$.

Equation (4) is similar to Equation (1) of Liivamägi et al. (2012). Since our spectroscopic sample is not dense enough to give a high number density of member galaxies in a cluster, we found only 224 galaxies for RSD corrections. The mean, median, and standard deviation of correction $\Delta d$ to comoving distances of these 224 galaxies are $1.29 \mathrm{Mpc}, 0.27 \mathrm{Mpc}$, and $1.73 \mathrm{Mpc}$. After applying these corrections, we again performed the clustering analysis with weights and found that the major results are not changed significantly. We again obtain $60 \mathrm{FoF}$ clusters in all, with Saraswati being the largest cluster having 386 galaxies in it, exactly the same as before. Reanalysis on the RSD-corrected LOWZ sample also concludes that RSD has a negligible effect on our clustering analysis.

This shows that the detection of the Saraswati Supercluster and other clusters is robust to redshift errors and peculiar motions in our data.

\section{Distribution of Known Clusters and Voids Near the Supercluster}

In the $\Lambda \mathrm{CDM}$ paradigm, clusters of galaxies trace the local extrema in the underlying distribution of matter-both dark and luminous, and since Saraswati is a particularly vast and overdense structure of galaxies, some clusters or groups of galaxies should be found at the highest density peaks. Moreover, in the cosmic web overdense regions are surrounded by almost empty voids. For quantifying more objectively the galaxy distribution in overdense (clusters/groups, filaments) and the underdense (voids) regions of the cosmic web around the Saraswati supercluster, we cross-match the galaxy distribution with the published catalogs of clusters and voids derived from SDSS data.

We compare the distribution of galaxies in our sample region with the clusters listed in SDSS-III cluster catalog of Wen et al. (2012; hereafter the WHL catalog). In the WHL catalog, the photometric redshifts of the BCG of each cluster are given. The spectroscopic redshifts are also listed but only if spectra are available in SDSS. We have used all available spectroscopic redshifts in SDSS-III for the BCGs of WHL galaxy clusters. For other clusters (small in number) whose BCG spectra are not available, we used photometric redshifts. In this way, we identified 238 WHL clusters in all within our analysis region. The WHL cluster catalog is $\approx 75 \%$ complete for clusters of $M_{200}>0.6 \times 10^{14} M_{\odot}$ and redshifts $z<0.42$, and $\approx 100 \%$ complete for clusters with $M_{200}>2 \times 10^{14} M_{\odot}$ and $z<0.5$ (Wen et al. 2012).

Out of the 238 WHL clusters within our analysis region there is a major concentration of 48 clusters within $90 \mathrm{Mpc}$ comoving distance from the center of Saraswati, and 43 of these are associated with the filament/wall-like main structure of the Saraswati supercluster (Figures 10 and 13). The data on these clusters are given in Table 1, listed in decreasing order of their mass $\left(M_{200}\right)$. The first cluster in the list is Abell 2631 $(z=0.277)$ and the second one is $\mathrm{ZwCl} 2341.1+0000$ $(z=0.269)$, both well-known clusters. We have further analyzed the properties of these WHL clusters below and used them to estimate the total mass and overdensity of Saraswati.

For the study of voids, we use the LOWZ void catalog of Nadathur (2016) based on the BOSS data of SDSS Data Release 11 (hereafter "Nadathur voids"). Nadathur voids are identified using the Voronoi Tessellation and Watershed algorithms. These are disjointed voids-independent underdense regions of space that do not overlap with each other. We have identified a total of 24 voids within our analysis region whose comoving radii range from $\approx 20 \mathrm{Mpc}$ to $\approx 86 \mathrm{Mpc}$.

Clusters and Voids-LOWZ Sample: the distribution of WHL clusters in and around Saraswati is shown in Figure 8 where clusters are plotted with star symbols. This figure shows that the number density of galaxy clusters within the Sarasawati region is much higher compared to other lower density regions. A similar result is obtained using the LBS galaxy sample discussed below. Figure 9 shows the distribution of voids within our analysis region. The circles (red dashed) show the voids, cross markers show the centers of voids and the radii of voids are the effective radii as given in the Nadathur void catalog. We can easily see that many voids surround the Sarasawati supercluster, which is expected because superclusters are always surrounded by voids.

Clusters and Voids-LBS Sample: similarly, the WHL clusters and Nadathur voids are plotted on the LBS galaxy sample, shown in Figures 10 and 11.

The high density of galaxies within Saraswati in both galaxy samples (LOWZ and LBS), high density of WHL clusters within Saraswati, and the presence of large voids surrounding it strongly support the physical existence of the Saraswati supercluster.

\section{Properties of the Saraswati Supercluster}

\subsection{Mass Distribution}

We have identified the Saraswati supercluster as a high density region of galaxies at mean redshift $z \sim 0.28$. A search through the Abell cluster catalog (Abell et al. 1989) shows that it is centered on the extremely rich (Abell richness class $R=3$ ), massive $\left(M_{500} \approx 10^{15} M_{\odot}\right)$, and hot $\left(T_{e}=8 \mathrm{KeV}\right)$ galaxy cluster Abell 2631 at redshift $z=0.277$. Abell 2631 is detected strongly in X-rays and also shows a significant Sunyaev-Zel'dovich decrement signal on the CMBR (Reese et al. 2012). We show below that the dominant Abell 2631 and a few more nearby massive clusters most likely form the dense, bound core of the Saraswati supercluster. Another unusual galaxy cluster $\mathrm{ZwCl} 2341.1+0000(z=0.269)$, a highly filamentary, multiply merging system, is located $\sim 45 \mathrm{Mpc}$ to the south in a filamentary spur joining the main Saraswati concentration. This one is the second-most massive cluster in the supercluster region. The faint, non-thermal synchrotron radio emission on a $\mathrm{Mpc}$ scale discovered in the $\mathrm{ZwCl} 2341.1$ +0000 cluster (Bagchi et al. 2002; van Weeren et al. 2009; Giovannini et al. 2010) is a clear signpost of the infall and merger dynamics of several galaxy groups during the first phase of the cluster formation process (Boschin et al. 2013). 
Table 1

WHL Clusters in and around the Saraswati Supercluster

\begin{tabular}{|c|c|c|c|c|c|c|c|c|}
\hline SrNo & $\begin{array}{l}\text { R.A. } \\
\text { (deg) }\end{array}$ & $\begin{array}{l}\text { Decl. } \\
\text { (deg) }\end{array}$ & Redshift & $r$ mag & $\begin{array}{c}R_{200} \\
(\mathrm{Mpc})\end{array}$ & $N_{200}$ & $\begin{array}{c}M_{200} \\
\left(10^{14} M_{\odot}\right)\end{array}$ & $\begin{array}{l}\text { Comv. Dist. } \\
\text { (Mpc) }\end{array}$ \\
\hline$\dagger 1$ & 354.41553 & 0.27137 & 0.2772 & 17.23 & 1.93 & 103 & 10.4544 & 0.000 \\
\hline 2 & 355.89862 & 0.33093 & 0.2694 & 17.93 & 1.62 & 79 & 7.4352 & 40.838 \\
\hline 3 & 356.59955 & 0.74942 & 0.2746 & 17.42 & 1.60 & 51 & 4.6789 & 44.331 \\
\hline$\dagger 4$ & 355.27872 & 0.30925 & 0.2768 & 17.21 & 1.36 & 32 & 2.9718 & 16.843 \\
\hline 5 & 354.40656 & -0.67781 & 0.2876 & 17.51 & 1.39 & 31 & 2.5886 & 43.159 \\
\hline 6 & 356.86499 & -0.15381 & 0.2639 & 17.91 & 1.33 & 37 & 2.4723 & 68.773 \\
\hline 7 & 350.62363 & -0.37294 & 0.2728 & 18.33 & 1.32 & 34 & 2.4673 & 75.977 \\
\hline 8 & 351.68036 & 1.13423 & 0.2774 & 18.28 & 1.37 & 30 & 2.4354 & 55.740 \\
\hline 9 & 351.88278 & 0.94281 & 0.2788 & 17.14 & 1.27 & 28 & 2.3992 & 51.391 \\
\hline 10 & 356.51950 & -0.18573 & 0.2665 & 17.62 & 1.28 & 27 & 2.1499 & 57.477 \\
\hline 11 & 357.56891 & 0.88373 & 0.2767 & 17.79 & 1.28 & 28 & 2.1138 & 62.379 \\
\hline 12 & 352.86020 & 0.61525 & 0.2739 & 17.84 & 1.20 & 26 & 1.7984 & 33.171 \\
\hline 13 & 357.83884 & 0.61691 & 0.2775 & 17.67 & 1.19 & 23 & 1.6145 & 66.884 \\
\hline 14 & 358.78690 & 0.75341 & 0.2789 & 17.87 & 1.07 & 24 & 1.4692 & 85.906 \\
\hline 15 & 354.03653 & -1.18347 & 0.2650 & 17.75 & 1.10 & 26 & 1.4580 & 54.053 \\
\hline 16 & 352.03851 & 0.18593 & 0.2768 & 17.38 & 1.12 & 16 & 1.3240 & 46.205 \\
\hline 17 & 356.45068 & -1.12771 & 0.2797 & 17.67 & 1.08 & 19 & 1.2245 & 49.083 \\
\hline 18 & 356.19455 & -0.08880 & 0.2674 & 17.98 & 1.05 & 17 & 1.1919 & 50.579 \\
\hline 19 & 354.56683 & 0.11680 & 0.2711 & 17.60 & 1.07 & 24 & 1.1810 & 23.271 \\
\hline 20 & 358.25555 & -0.23089 & 0.2779 & 17.86 & 1.01 & 10 & 1.0581 & 75.367 \\
\hline 21 & 356.01273 & 0.22644 & 0.2714 & 18.34 & 1.00 & 18 & 1.0368 & 37.667 \\
\hline 22 & 356.86264 & 0.52773 & 0.2729 & 17.74 & 1.00 & 12 & 1.0255 & 50.122 \\
\hline 23 & 353.99393 & -0.48434 & 0.2700 & 17.79 & 0.99 & 15 & 1.0180 & 31.727 \\
\hline 24 & 356.32980 & -0.05331 & 0.2665 & 18.29 & 0.98 & 19 & 1.0080 & 54.668 \\
\hline$\dagger 25$ & 355.10202 & -0.09299 & 0.2761 & 18.09 & 0.98 & 14 & 0.9881 & 15.627 \\
\hline 26 & 358.93271 & -0.00393 & 0.2752 & 17.95 & 0.98 & 16 & 0.9868 & 87.932 \\
\hline 27 & 353.62631 & -0.98501 & 0.2772 & 17.79 & 0.96 & 9 & 0.9502 & 28.827 \\
\hline 28 & 355.43375 & -0.67539 & 0.2680 & 17.78 & 0.94 & 12 & 0.9107 & 43.606 \\
\hline 29 & 357.93292 & 0.56078 & 0.2703 & 18.01 & 0.95 & 17 & 0.9083 & 72.542 \\
\hline 30 & 357.23520 & -0.89120 & 0.2793 & 17.72 & 0.93 & 9 & 0.9034 & 59.979 \\
\hline$\dagger 31$ & 354.97913 & -0.43282 & 0.2769 & 17.53 & 0.94 & 13 & 0.8991 & 17.552 \\
\hline 32 & 351.02548 & 0.36311 & 0.2780 & 17.90 & 0.93 & 12 & 0.8825 & 66.039 \\
\hline 33 & 352.66733 & 0.60940 & 0.2757 & 17.66 & 0.92 & 14 & 0.8295 & 34.962 \\
\hline 34 & 356.67987 & 0.83429 & 0.2637 & 17.89 & 0.93 & 15 & 0.8265 & 67.370 \\
\hline 35 & 351.58939 & 0.16173 & 0.2870 & 18.15 & 0.88 & 12 & 0.7956 & 66.786 \\
\hline 36 & 353.87994 & -0.88882 & 0.2974 & 17.95 & 0.93 & 11 & 0.7842 & 79.549 \\
\hline 37 & 352.81964 & 0.72678 & 0.2812 & 18.34 & 0.93 & 11 & 0.7782 & 35.747 \\
\hline 38 & 352.17755 & 0.82753 & 0.2784 & 17.42 & 0.92 & 9 & 0.7584 & 45.116 \\
\hline$\dagger 39$ & 353.87866 & -0.53116 & 0.2764 & 18.27 & 0.84 & 10 & 0.7452 & 18.973 \\
\hline 40 & 356.76236 & -0.08544 & 0.2655 & 17.61 & 0.88 & 11 & 0.7327 & 63.065 \\
\hline 41 & 358.12292 & 0.60623 & 0.2698 & 17.91 & 0.88 & 12 & 0.7018 & 76.620 \\
\hline 42 & 355.63776 & -0.28874 & 0.2770 & 18.30 & 0.86 & 11 & 0.6924 & 26.124 \\
\hline 43 & 358.60349 & 0.81066 & 0.2805 & 18.30 & 0.76 & 12 & 0.6835 & 83.396 \\
\hline 44 & 351.32236 & 0.27741 & 0.2767 & 18.33 & 0.87 & 9 & 0.6553 & 60.071 \\
\hline 45 & 351.95999 & -0.64258 & 0.2856 & 18.08 & 0.85 & 8 & 0.6424 & 60.422 \\
\hline 46 & 358.44800 & 0.81641 & 0.2787 & 17.80 & 0.81 & 8 & 0.6040 & 79.441 \\
\hline 47 & 355.18701 & 0.31234 & 0.2982 & 19.15 & 0.86 & 13 & 0.5994 & 79.790 \\
\hline 48 & 357.44406 & 0.64598 & 0.2705 & 17.84 & 0.63 & 8 & 0.5988 & 63.779 \\
\hline
\end{tabular}

Note. Data on 48 galaxy clusters listed in WHL catalog, within $90 \mathrm{Mpc}$ comoving distance from the center of Saraswati supercluster. Abell 2631, the most massive cluster and first in the list, is considered the center of the supercluster. The columns are as follows: (1) cluster number; $(2,3)$ R.A. and decl. of the BCG; (4) redshift; (5) r-band magnitude of the BCG; $(6,7,8) R_{200}, N_{200}$ (richness parameter), and $M_{200}$; (9) Comoving distance between Abell 2631 and the cluster's BCG. Clusters are arranged in decreasing order of their mass and those marked with a $\dagger$ sign are the five clusters within the bound core of the supercluster.

The optical images of clusters Abell 2631 and ZWCl 2341.1 +0000 are shown in Figure 12.

The main body of Saraswati supercluster contains at least 43 massive clusters/groups of galaxies identified from the WHL catalog. The total mass of a supercluster is indicated by the number of massive clusters it contains (Chon et al. 2014). The total bound halo mass of these 43 clusters is estimated to be $\approx 2 \times 10^{16} M_{\odot}$, as explained below. From the known masses of clusters, through X-ray and weak-lensing scaling relations, and their richness, Wen et al. (2012) found a correlation between the virial mass $M_{200}$ and the cluster richness parameter $R_{L *}$ of their WHL clusters, given by

$$
\log M_{200}=(-1.49 \pm 0.05)+(1.17 \pm 0.03) \log R_{L *},
$$

where $M_{200}$ is in units of $10^{14} M_{\odot}$.

Using this relation, we estimate the mass of each cluster within $r_{200}$ (see Table 1). However, a significant amount of matter must lie beyond the virial radius of a cluster, which 


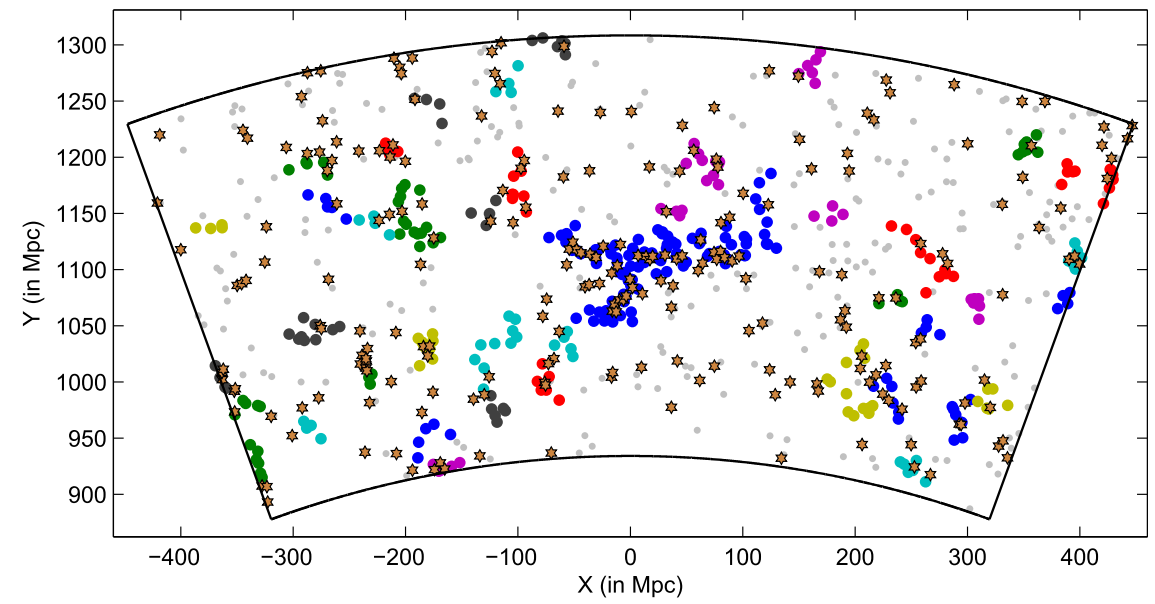

Figure 8. Here we plot the WHL clusters (denoted by stars) along with FoF clusters identified with LOWZ galaxy data. The high density of clusters near the Saraswati supercluster is very clear.

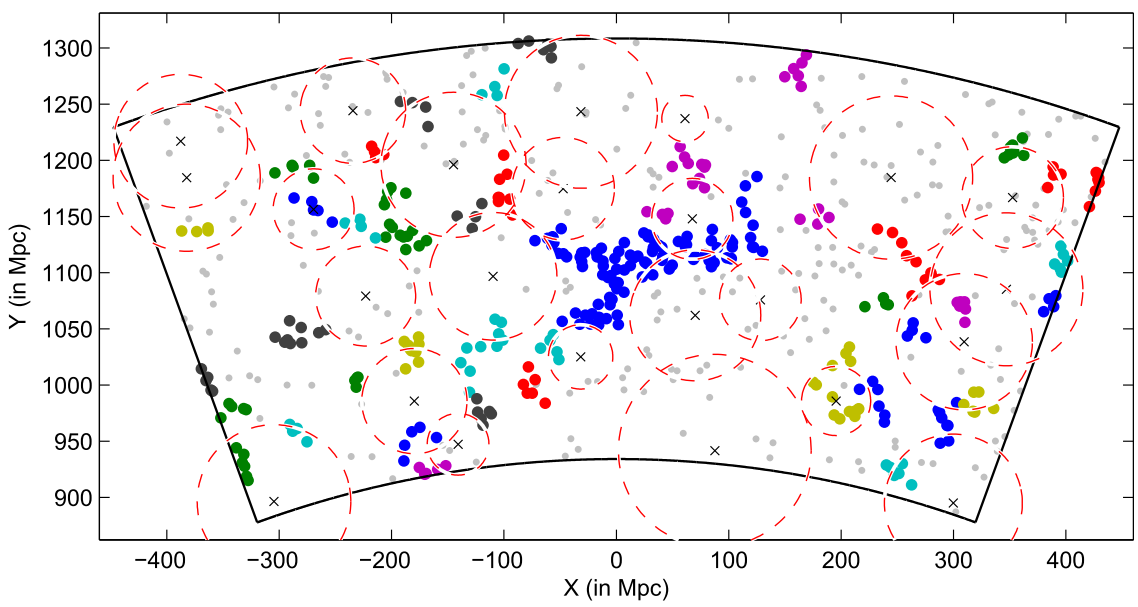

Figure 9. Voids distribution in LOWZ subsample. The FoF clusters are shown in various colors. Red dashed circles are voids in projection, black crosses are void centers, and the radii of circles are equal to the effective radii of voids as given in the Nadathur void catalog.

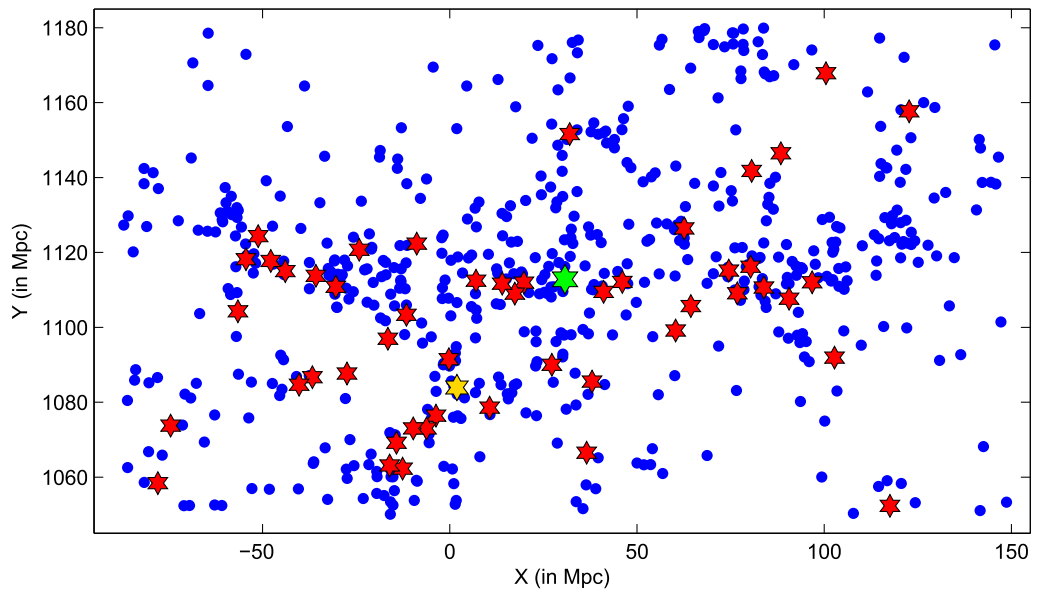

Figure 10. In this figure we show galaxy clusters near the Saraswati supercluster. All cataloged WHL Clusters (red stars) are plotted over the galaxies from the LBS sample (blue dots). The location of the most massive cluster Abell 2631 is shown with a green star and the second-most massive cluster ZwCL $2341.1+0000$ by a yellow star (see Figure 12 and Table 1).

needs to be accounted for. Therefore, this estimate of virial mass $M_{200}$ is further scaled up to calculate the bound halo mass $M_{\text {halo }} \approx M_{5.6}$ of each cluster; where $M_{5.6}$ denotes the mass inside $r_{5.6}$, the radius within which the mass overdensity is 5.6 times the critical density of the universe. Several studies find that the bound halo mass of a cluster is close to $M_{5.6} \sim 2.2 \times M_{200}$ (Busha et al. 2005; Rines \& Diaferio 2006; Rines et al. 2013). This way we estimate the total halo mass of 43 main clusters to be $\sim 2 \times 10^{16} M_{\odot}$. We point out that this is strictly a lower limit as the full extent of the Saraswati supercluster is not known yet 


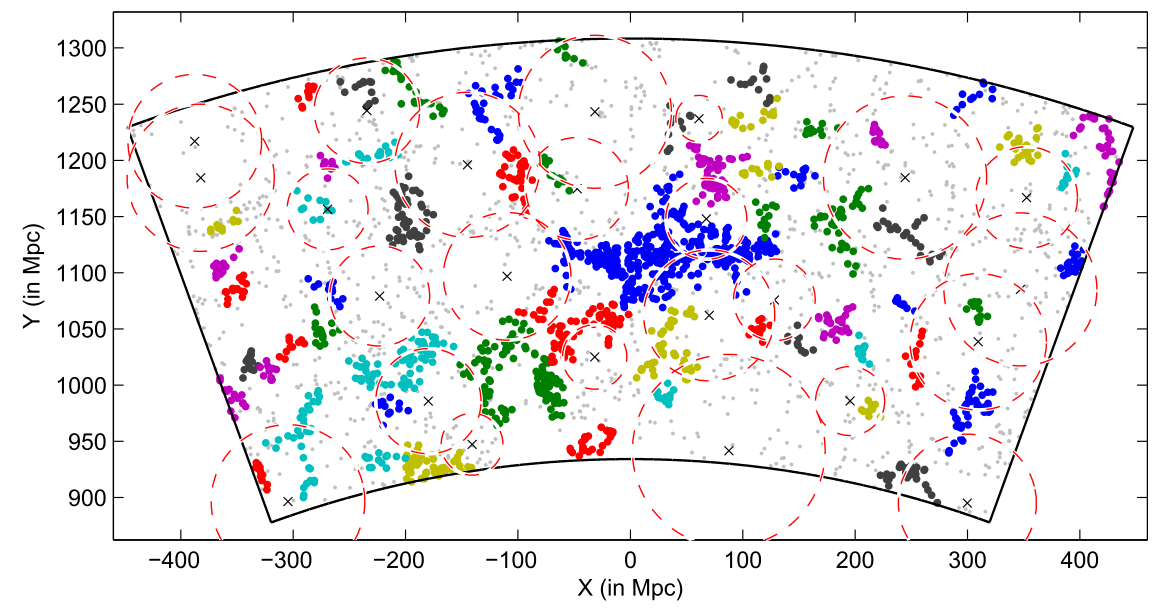

Figure 11. In this figure we show voids in the Saraswati supercluster region. Cataloged voids (Nadathur 2016) are plotted over the LBS galaxy sample. The FoF clusters are shown in various colors. Red dashed circles are voids in projection, black crosses are void centers, and the radii of circles are equal to the effective radii of voids as given in the Nadathur void catalog.
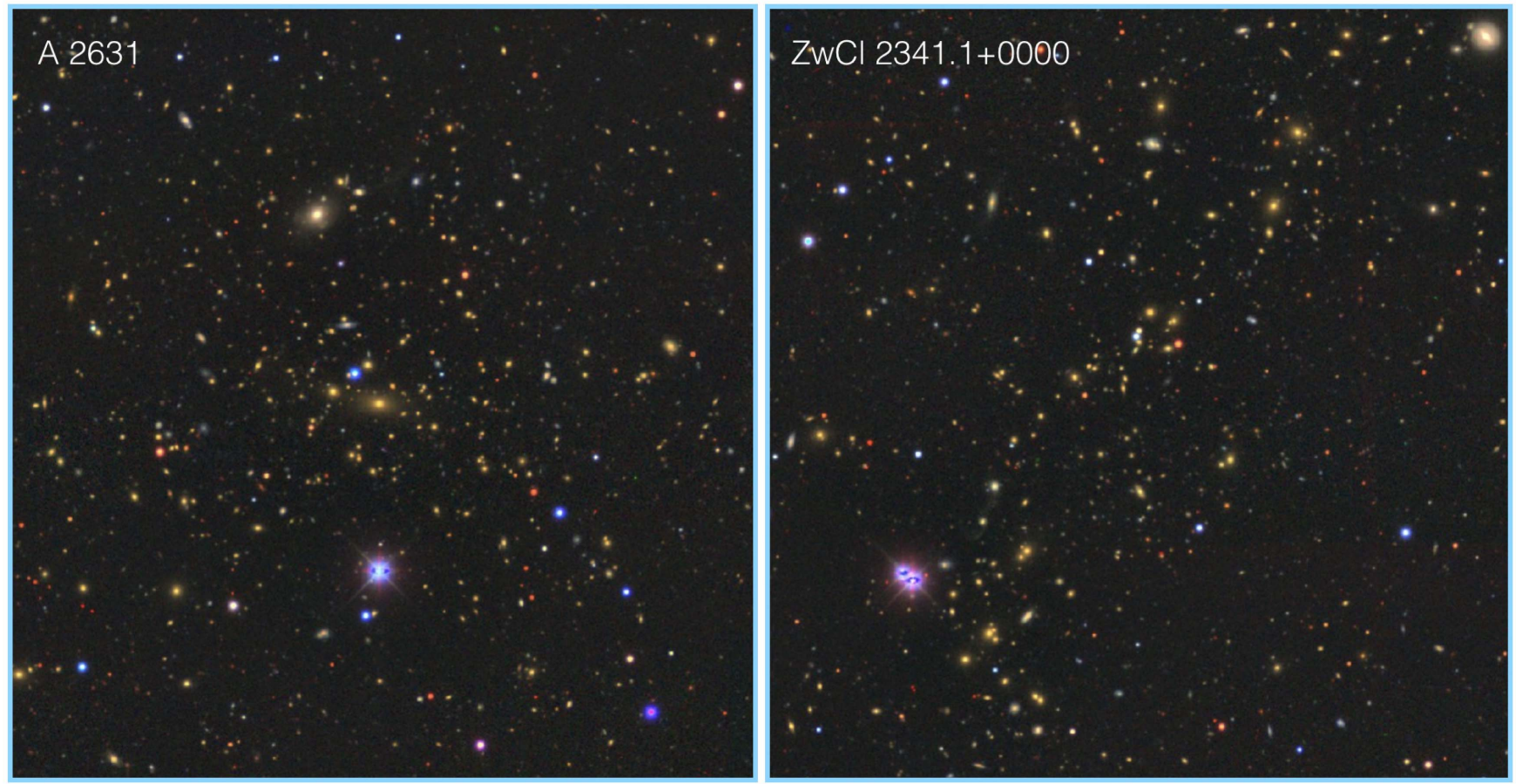

Figure 12. Optical images of the two most massive clusters of the Saraswati supercluster; with the relaxed-type Abell $2631(z=0.277)$ on left and the highly filamentary $\mathrm{ZWCl} 2341.1+0000(z=0.269)$ on the right, taken from the DECam legacy survey (DECaLS). Each image is $\approx 10^{\prime} \times 10^{\prime}$ across, corresponding roughly to $2.5 \mathrm{Mpc}$ on a side.

(due to the decl. limit of SDSS on Stripe 82), and some fraction of total mass is still unaccounted for, which is not bound to the virializing dark matter halos.

Figure 13 (upper) plots the $3 \mathrm{D}$ distribution $(X, Y, Z$ comoving coordinates) of 43 clusters in Saraswati supercluster, spanning $\sim 200 \mathrm{Mpc}$ across, and shows low-mass clusters surround the massive clusters like irregular halos. In this figure the radius of a sphere (cluster) is proportional to its $r_{200}$. Colors represent the masses of the clusters. The largest dark red sphere is the most massive Abell $2631\left(M_{200} \approx 10^{15} M_{\odot}\right.$; Reese et al. 2012) and small blue spheres show the least massive clusters. Significantly, 23 among these 43 clusters are very massive, having virial masses $M_{200}>1 \times 10^{14} M_{\odot}$ (Table 1$)$. This implies an unusually high-mass concentration in massive clusters within the supercluster region. In $\Lambda \mathrm{CDM}$ cosmology, the expected number of dark matter halos (Sheth et al. 2001; with mass $\left.M_{200}>10^{14} M_{\odot}\right)$ within the Saraswati supercluster volume (estimated below), at $z \sim 0.28$, is only $\approx 2$.

\subsection{Bounded Region of the Saraswati-TurnAround Radius}

While a supercluster of the scale of Saraswati is unlikely to be in a state of dynamical equilibrium with well-defined boundaries, one can still ask whether or not it is gravitationally bound. We now try to estimate how much fraction of the supercluster is possibly gravitationally bound.

Although Saraswati is more planar than spherical, we nevertheless apply the widely used spherical collapse model in which the turnaround radius $R_{\mathrm{ta}}$ (the radius of the shell separating infalling material from the outward expansion of the 

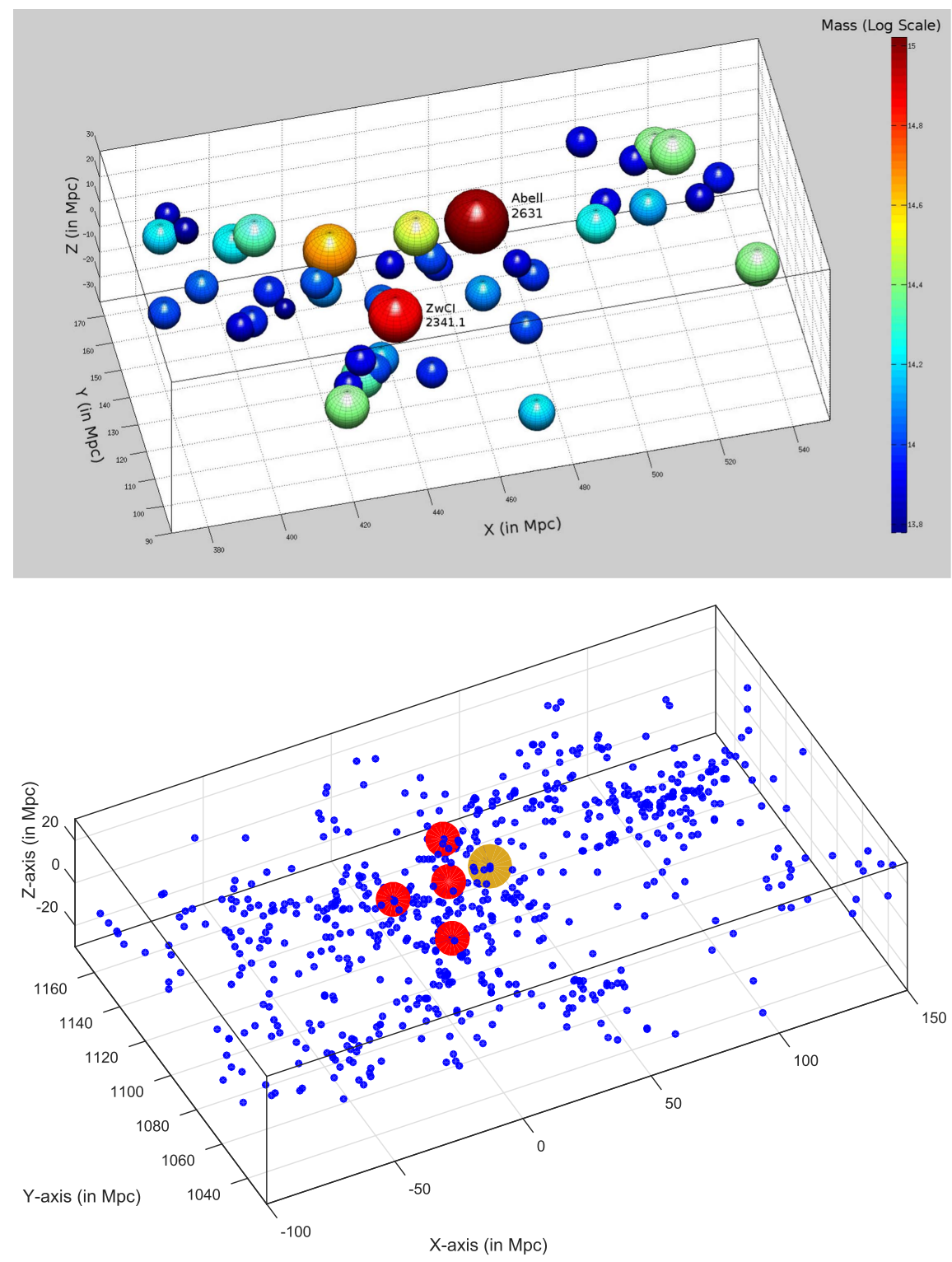

Figure 13. Upper: this figure shows the 3D distribution of the wall-like structure of the Saraswati supercluster, mainly comprising 43 known clusters of galaxies, here represented by spheres. The radius of each cluster is proportional to its virial radius $r_{200}$. Colors represent the masses of clusters (in $\log \left(M_{200}\right)$ scale shown with the color bar). The dark red sphere represents the most massive and rich galaxy cluster Abell 2631 and bright red the next massive one, ZwCl $2341.1+0000$, and subsequently orange, lemon yellow, green, etc. Dark blue spheres show the least massive clusters. Lower: 3D distribution of the galaxies and some of the most massive clusters near the Abell 2631 cluster are shown. Small blue circles are the LBS galaxies, the large golden circle represents Abell 2631, and the other big red circles are the next four most massive clusters of Saraswati supercluster.

Hubble flow (Peebles 1980; Padmanabhan 1993; Busha et al. 2003; Pavlidou \& Tomaras 2014) for a mass $M$ is

$$
R_{\mathrm{ta}}=\left(\frac{3 G M}{\Lambda c^{2}}\right)^{\frac{1}{3}},
$$

or

$$
R_{\mathrm{ta}}=\left(\frac{3 G M}{H_{o}^{2} \Omega_{\Lambda}}\right)^{\frac{1}{3}},
$$

where $\Lambda=\left(8 \pi G \rho_{\Lambda} / c^{2}\right)$ is the cosmological constant and the dark energy density is $\rho_{\Lambda}$. Since the total halo mass of each cluster cannot be independently calculated, we use results based on numerical simulations. As before, total bound halo mass of a component cluster is taken to be $M_{5.6} \approx 2.2 \times M_{200}$ following (Busha et al. 2005; Rines \& Diaferio 2006; Rines et al. 2013), and thus we obtain the sum of masses of 43 clusters as the total mass of Saraswati, which is $M \approx 2 \times 10^{16} M_{\odot}$. This is strictly a lower limit because the mass of matter (dark-matter + galaxies) dispersed within the intercluster space is not included. Below we have tried to correct for the extra mass.

Using this total mass in Equation (7) above, we obtain $R_{\mathrm{ta}} \approx 30$ Mpc, or only about one-third of its $\approx 100 \mathrm{Mpc}$ physical radius. This suggests that the entire Saraswati supercluster cannot be a gravitationally bound structure. Further, by integrating in spherical shells the enclosed galaxy cluster masses up to a certain radius $R$ and comparing that with $R_{\mathrm{ta}}$ (Figure 14), we infer that only the mass within the radius $R \sim 20 \mathrm{Mpc}$ is possibly gravitationally bound and could have reached turnaround. Four high-mass galaxy clusters, each of mass $M_{200} \sim 10^{14} M_{\odot}$, and the most massive Abell $2631\left(M_{200} \gtrsim 10^{15} M_{\odot}\right.$; Reese et al. 2012), near the center, comprise this bound core (Table 1), as one would expect from the 


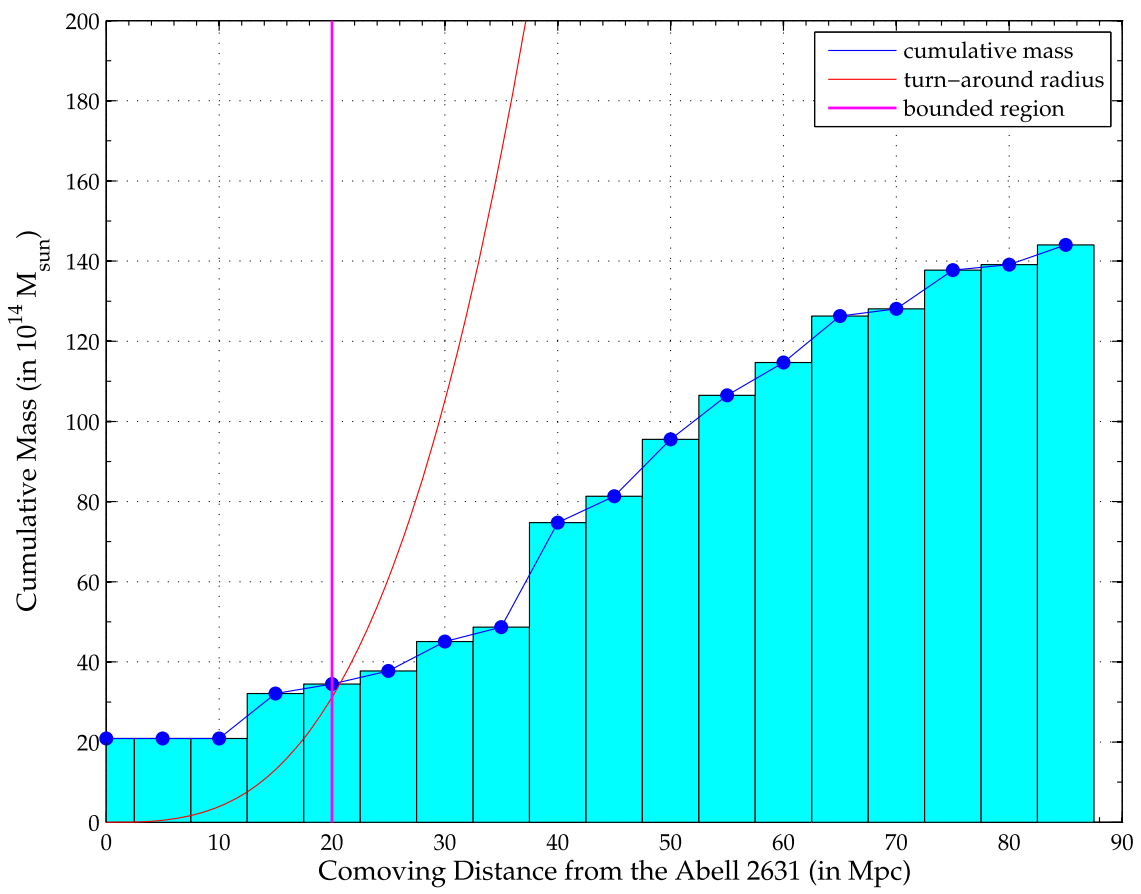

Figure 14. The radial mass distribution of WHL clusters in the Saraswati supercluster up to $R \approx 90 \mathrm{Mpc}$ radius. The $x$-axis is the radial distance $(R)$ from Abell 2631 (the most massive galaxy cluster in the core of the Saraswati supercluster) and the $y$-axis is the cumulative bound halo mass of the WHL clusters within the sphere of radius $R$ (bin size $\sim 5 \mathrm{Mpc}$ ). The red curve is the theoretical turnaround radius (Equation (7)). The blue curve shows the cumulative mass distribution. Five clusters are inside the turnaround curve and 38 are outside it. The clusters within the turnaround radius are altogether bound while the clusters beyond it are unbound. The bounded region has a comoving radius of $\approx 20 \mathrm{Mpc}$ (magenta line).

gravitational collapse model. The aggregate mass of this dense, bound core is at least $4 \times 10^{15} M_{\odot}$, or about $20 \%$ of the mass of the entire supercluster. Even neglecting the (unknown) small mass of matter dispersed within the intercluster space, this gives a lower bound to the core overdensity of $\rho_{\text {core }} / \rho_{\mathrm{cz}}=1.4$ and $\rho_{\text {core }} / \rho_{m}=3.12$. Here $\rho_{\text {core }}, \rho_{m}$, and $\rho_{\text {cz }}$ are the density of the bound core of Saraswati, the mean matter density of the universe at $z=0.28$, and the critical density of the universe at $z=0.28$, respectively. According to the definition of a supercluster proposed by Chon et al. (2015), superclusters are structures defined based on an overdensity criterion that selects only those objects that will collapse in the future, including those that are at a turnaround in the present epoch. Therefore, if we adopt this definition of a supercluster, Saraswati indeed qualifies as being a supercluster.

\section{Density Contrast of the Supercluster}

Here we calculate the density contrast of the Saraswati supercluster. As we already have the halo mass information of all 43 clusters, we now need to estimate the volume $(V)$ of Saraswati to calculate its mass density. Since Saraswati is a walllike extended structure we do not assume a spherical volume because that would largely overestimate $V$ and give a much smaller density contrast. Therefore, to obtain $V$ we calculate the volume of the Convex Hull of the 43 clusters (set of points) within Saraswati. A Convex Hull of a set of points $\boldsymbol{P}$ in 3D Euclidean space that is the convex surface (envelope) of the minimum possible volume on $\boldsymbol{P}$. We use the MATLAB Qhull algorithm (Barber et al. 1996) to construct the Convex Hull and to calculate its volume. In this way, we calculate the comoving volume of Saraswati as $V \approx 2.05 \times 10^{5} \mathrm{Mpc}^{3}$. Now the corresponding matter density contrast $\delta$ is

$$
\delta=\frac{\rho_{\mathrm{SS}}}{\rho_{m}}-1=\left(\frac{\rho_{\mathrm{SS}}}{\rho_{c}} \frac{1}{\Omega_{M}}\right)-1,
$$

where $\rho_{m}$ is the background matter density at redshift $z=0.28$, $\rho_{c}$ is the critical density at the present epoch, and $\rho_{\mathrm{SS}}$ is the comoving mass density of the Saraswati supercluster. For the supercluster mass we use the total bound halo mass of 43 clusters, estimated above to be $\approx 2 \times 10^{16} M_{\odot}$, and use that to calculate $\rho_{\mathrm{SS}}$. This gives an average matter density contrast $\delta=1.62\left(\rho_{\mathrm{SS}} / \rho_{m}=2.62\right)$ and $\rho_{\mathrm{SS}} / \rho_{c}=1.17$ for the supercluster. Both these numbers fall short of the minimum density $\rho / \rho_{c}>2.36$ and $\rho / \rho_{m}>7.86$ necessary if the entire supercluster is to remain gravitationally bound at present, and also in the distant future (Nagamine \& Loeb 2003; Dünner et al. 2006). We point out that at the present epoch the largest known gravitationally bound, virialized structures are the galaxy clusters spanning a few Mpc across and masses $\sim 10^{14-15} M_{\odot}$. If the space between the clusters of the Saraswati supercluster is uniformly filled with matter at background density $\rho_{m}$, then its total mass will be $\approx 4 \times 10^{16} M_{\odot}$, giving a higher density contrast $\delta \approx 3$ and $\rho_{\mathrm{SS}} / \rho_{m} \approx 4$.

\section{Discussion and Conclusions}

Showing a significant mass overdensity on $\approx 200 \mathrm{Mpc}$ scale, the Saraswati supercluster clearly stands out on the sky as an especially rare, and possibly among the largest prominent density enhancements found at medium-high redshift epochs $0.2<z<0.6$. Only very few such massive superclusters are known at present. Observing such massive, large-scale structures 
at early cosmic aeons has major implications for cosmological studies, as the number of highest density peaks represented by clusters and superclusters and their growth function places strong constraints on the nature of the primordial fluctuation field and the dark energy equation of state, which connects pressure $(P)$ to energy density $\left(\rho_{\lambda}\right) ; P=w(z) \rho_{\lambda}$ (Sahni \& Starobinsky 2000; Frieman et al. 2008; Sheth \& Diaferio 2011; Park et al. 2012). Further, to ascertain if Einstein's general relativity correctly describes the formation of such large structures, testing it across a wide range of physical scales and mass densities is necessary, and superclusters like Saraswati are powerful probes of gravity in its extreme large-scale, weak-field limit (Jain \& Khoury 2010). Numerical simulations are also very important to test and calibrate the competing cosmological models as applied to the present structures (Park et al. 2012).

Interestingly, the redshift epoch of the Saraswati complex is near $z=\left(\Omega_{m} / \Omega_{\Lambda}\right)^{1 / 3 w}-1 \approx 0.37$ (for $w=-1$ ) when energy density in matter $\left(\Omega_{m}\right)$ and dark energy $\left(\Omega_{\Lambda}\right)$ became almost equal and the growth of large-scale perturbations virtually ceased due to the accelerated expansion of the universe (Frieman et al. 2008). This gives rise to an important question: Did the major growth of this supercluster take place much before the dark energy dominated era $(z \sim 0.7)$, or did it happen fairly recently, when it entered into a quasi-linear $\left(\delta_{m}=O(0.1)\right)$ growth regime? A large-scale structure this massive evolves very slowly and therefore it may reflect the whole history of galaxy formation and the primordial initial conditions that have "seeded" it. The mass and spatial scale of the present superstructure is comparable to that of the Sloan Great Wall (Gott et al. 2005; 200-400 Mpc) and the Shapley Concentration (Raychaudhury 1989; Bardelli et al. 2000; Proust et al. 2006; $200 \mathrm{Mpc}$ ), two of the largest and most massive structures found in the nearby universe. Other important questions that arise from this work are as follows. By what process have these extremely large and overdense cosmic structures formed and is their existence in accord with a homogeneous, isotropic universe with primordial Gaussian random phase density fluctuations as predicted by inflation?

The physical mechanism of supercluster formation is still not well understood. The probability of massive Saraswati-like superclusters arising from tiny $(\delta(\rho) / \rho<0.1)$ perturbations in the primordial Gaussian random density field is very small (Padmanabhan 1993). Therefore, significant galaxy concentrations developing on the scale of $\sim 200 \mathrm{Mpc}$ or more are puzzling, which might challenge the belief that the universe becomes (statistically) homogeneous and isotropic on scales $\gtrsim 100 h^{-1} \mathrm{Mpc}$ (Sarkar et al. 2009). These massive structures provide excellent model-independent tests of general relativity on the largest scales. For example Park et al. (2012) have identified only a few such extreme-scale superclusters (size $\sim 200-400 \mathrm{Mpc}$ ) in their Horizon Run 2 simulation covering $7.2^{3} h^{-3} \mathrm{Gpc}^{3}$ volume. They conclude that an initially homogeneous isotropic universe with primordial Gaussian random phase density fluctuations growing in accordance with general relativity, can explain the richness and size of the observed large-scale structures in SDSS. More recently another large, galactic-wall-like structure spanning $\sim 250 \mathrm{Mpc}$ at $z \sim 0.47$ was identified in the BOSS survey (Lietzen et al. 2016). On the other hand, Sheth \& Diaferio (2011) advocate that the formation of even a few extremely large and massive structures like the Sloan Great Wall in the local universe will result in tension with Gaussian initial conditions.
Dark energy (either a "cosmological constant" or some other form) appears to dominate the present universe, which not only alters its expansion rate but also affects the evolution of perturbations in the density of matter, slowing down the gravitational collapse of material in recent times (Frieman et al. 2008). A powerful probe of dark energy, and how it affects the time evolution of the gravitational potential of superclusters and voids, is provided by the observation of secondary temperature anisotropies in the cosmic microwave background (CMB) imprinted via the late-time integrated Sachs-Wolfe (and the Rees-Sciama effect in nonlinear regime; ISW+RS) effect (Nishizawa 2014). The existence of large-scale superclusters and voids of $\gtrsim 100 \mathrm{Mpc}$ size at $z \sim 0.5$ is implied by correlated CMB temperature fluctuations of $|\Delta T| \approx 10 \pm 2 \mu K$ ascribed to the late-time ISW+RS effect (Granett et al. 2008; Inoue et al. 2010; Giannantonio et al. 2012). The significance of such observations is still under scrutiny, but if confirmed they appear to be in tension with $\Lambda \mathrm{CDM}$ predictions, e.g., (Nadathur et al. 2012; Cai et al. 2014; Nadathur \& Crittenden 2016). For the Saraswati supercluster we try to obtain its ISW + RS signal, using its matter density contrast $\delta=1.62$ and effective comoving radius $r_{c}=[3 V / 4 \pi]^{1 / 3} \approx 40 \mathrm{Mpc}$. In the linear growth regime we obtain $\Delta T \approx 1.6 \mu K$ on $\approx 6.5$ scale, which is smaller than some present measurements (Granett et al. 2008; Inoue et al. 2010; Giannantonio et al. 2012). However, our estimation is uncertain at present owing to our lack of detailed knowledge about the shape and depth of gravitational potential of this supercluster and its time evolution. We highlight that finding some more extremely massive, overdense structures like Saraswati, the Shapley Concentration, and the Sloan/BOSS Great Wall at higher redshifts $(z \approx 0.3-1)$, in the dark energy dominated era, and measuring their ISW + RS and Sunyaev-Zel'dovich imprint on the CMBR sky will test the competing cosmological models and provide an alternative window into dark energy.

A possible clue to the formation of this large galactic superstructure is provided by the detection of huge voids of $\sim 40-170 \mathrm{Mpc}$ diameter observed around the main wall-like overdense structure of Saraswati. In $\Lambda$ CDM cosmology, space within voids (typically $\delta(\rho) / \bar{\rho} \sim-0.8$; Colberg et al. 2005) expands faster than the background Hubble expansion and thus matter inside voids will have an outward component of peculiar velocity away from the void centers (Padilla et al. 2012). Hence, matter within voids will be swept up into dense sheets or pancakes, which intersect one another, forming long galaxy filaments of the cosmic web separating neighboring voids. The overwhelmingly large negative pressure of dark energy $\left(P=w \rho_{\Lambda}, w=-1\right.$ with $\rho_{\Lambda} / \rho_{m} \sim 10-20$ inside void) and gravitational attraction of the surrounding mass distribution together govern the structure and dynamics of large-scale voids and possibly superclusters. This effect will be most pronounced when dark energy becomes dominant over matter at late times, around $z \sim 0.37$ for $w=-1$. We believe that this process might have played an important role in the evolution of nearby largescale structures like Saraswati, the Shapley Supercluster, or the Sloan/BOSS Great Wall. Recently, repulsion from a pronounced void in galaxy distribution (the "dipole repeller") has been attributed to the observed flow of nearby galaxies, converging toward a single attractor associated with the Shapley Supercluster (Hoffman et al. 2017), thus providing support to the model.

In the future, the Saraswati supercluster and its environs in Stripe 82 region must be surveyed in greater depth with more 
galaxy redshifts taken on a wider scale for a better understanding of what physical processes were involved in the growth of such enormous cosmic structures in the distant universe. It also offers exciting possibilities for gravitational weak-lensing mapping of dark matter distributed in clusters, filaments, and voids (Vikram et al. 2015), and for Sunyaev-Zel'dovich imaging of the hot, baryonic matter via its characteristic imprint on the cosmic microwave background photons. This region is also a prime target for future deep surveys in X-rays, from the hot gas trapped in the numerous overdense collapsing structures (clusters) and from the mass-accreting super massive black holes (active galactic nuclei), and for deep radio observations of non-thermal shocks in the magnetized cosmic web (Kale et al. 2016). This supercluster region may contain large amounts of warm-hot intergalactic medium (WHIM), which might be detected via its spectral signatures in soft X-rays and UV. These observations will open a new window on the study of large-scale structures in the universe.

We thank the referee for the useful comments. S.S. acknowledges support from a PhD studentship at IISER (Pune). J.B., J.J., and P.D. gratefully acknowledge generous support from the IndoFrench Center for the Promotion of Advanced Research (Centre Franco-Indien pour la Promotion de la Recherche Avanćee) under programme no. 5204-2. J.J. acknowledges generous support from IUCAA's Associateship Program. Funding for SDSS-III has been
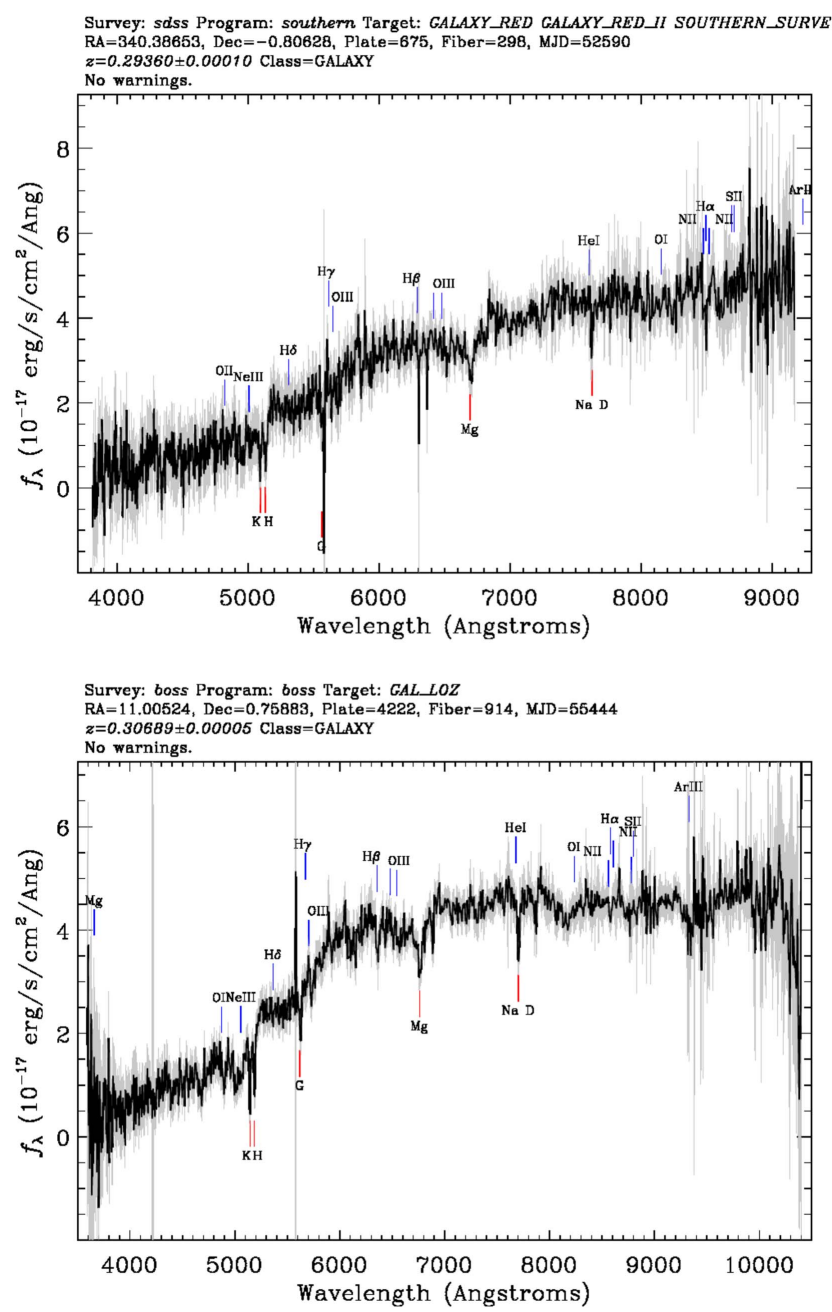

provided by the Alfred P. Sloan Foundation, the Participating Institutions, the National Science Foundation, and the U.S. Department of Energy Office of Science. The SDSS-III Web site is http://www.sdss3.org/. SDSS-III is managed by the Astrophysical Research Consortium for the Participating Institutions of the SDSS-III Collaboration including the University of Arizona, the Brazilian Participation Group, Brookhaven National Laboratory, Carnegie Mellon University, University of Florida, the French Participation Group, the German Participation Group, Harvard University, the Instituto de Astrofisica de Canarias, the Michigan State/Notre Dame/JINA Participation Group, Johns Hopkins University, Lawrence Berkeley National Laboratory, Max Planck Institute for Astrophysics, Max Planck Institute for Extraterrestrial Physics, New Mexico State University, New York University, Ohio State University, Pennsylvania State University, University of Portsmouth, Princeton University, the Spanish Participation Group, University of Tokyo, University of Utah, Vanderbilt University, University of Virginia, University of Washington, and Yale University.

\section{Appendix}

The spectra of a few galaxies from our sample are shown in the figure below.
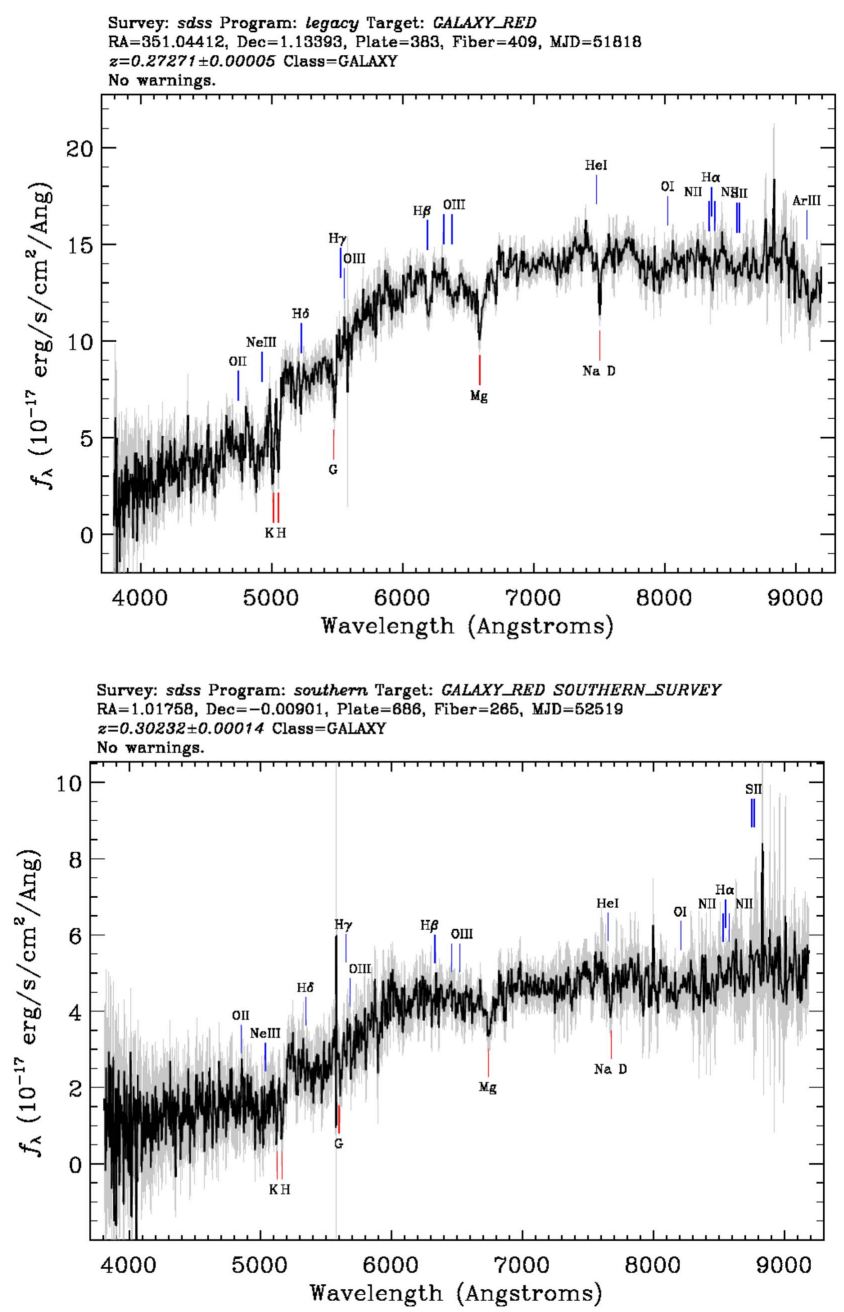

Figure 15. Spectra of four galaxies from our samples, taken from SDSS. The header of each spectra gives the sky position of the galaxy and its redshift with errors. 


\section{References}

Abell, G. O. 1961, AJ, 66, 607

Abell, G. O., Corwin, H. G., Jr., \& Olowin, R. P. 1989, ApJS, 70, 1

Ahn, C. P., Alexandroff, R., Allende Prieto, C., et al. 2012, ApJS, 203, 21

Alam, S., Albareti, F. D., Allende Prieto, C., et al. 2015, ApJS, 219, 12

Bagchi, J., Enßlin, T. A., Miniati, F., et al. 2002, NewA, 7, 249

Barber, C. B., Dobkin, D. P., \& Huhdanpaa, H. 1996, ACM Trans. Math. Softw., 22, 469

Bardelli, S., Zucca, E., Zamorani, G., Moscardini, L., \& Scaramella, R. 2000, MNRAS, 312, 540

Blanton, M. R., \& Roweis, S. 2007, AJ, 133, 734

Bond, J. R., Kofman, L., \& Pogosyan, D. 1996, Natur, 380, 603

Boschin, W., Girardi, M., \& Barrena, R. 2013, MNRAS, 434, 772

Busha, M. T., Adams, F. C., Wechsler, R. H., \& Evrard, A. E. 2003, ApJ, 596, 713

Busha, M. T., Evrard, A. E., Adams, F. C., \& Wechsler, R. H. 2005, MNRAS, 363, L11

Cai, Y.-C., Neyrinck, M. C., Szapudi, I., Cole, S., \& Frenk, C. S. 2014, ApJ, 786, 110

Chon, G., Böhringer, H., Collins, C. A., \& Krause, M. 2014, A\&A, 567, A144

Chon, G., Böhringer, H., \& Zaroubi, S. 2015, A\&A, 575, L14

Colberg, J. M., Sheth, R. K., Diaferio, A., Gao, L., \& Yoshida, N. 2005, MNRAS, 360, 216

de Vaucouleurs, G. 1956, VA, 2, 1584

Dünner, R., Araya, P. A., Meza, A., \& Reisenegger, A. 2006, MNRAS, 366, 803

Einasto, M., Einasto, J., Tago, E., Dalton, G. B., \& Andernach, H. 1994, MNRAS, 269, 301

Einasto, J. 2014, Dark Matter and Cosmic Web Story (Singapore: World Scientific)

Frieman, J. A., Turner, M. S., \& Huterer, D. 2008, ARA\&A, 46, 385

Giannantonio, T., Crittenden, R., Nichol, R., \& Ross, A. J. 2012, MNRAS, 426,2581

Giovannini, G., Bonafede, A., Feretti, L., Govoni, F., \& Murgia, M. 2010, A\&A, 511, L5

Gott, J. R., III, Jurić, M., Schlegel, D., et al. 2005, ApJ, 624, 463

Granett, B. R., Neyrinck, M. C., \& Szapudi, I. 2008, ApJL, 683, L99

Hoffman, Y., Pomarède, D., Tully, R. B., \& Courtois, H. M. 2017, NatAs, 1, 36
Huchra, J. P., \& Geller, M. J. 1982, ApJ, 257, 423

Inoue, K. T., Sakai, N., \& Tomita, K. 2010, ApJ, 724, 12

Jain, B., \& Khoury, J. 2010, AnPhy, 325, 1479

Jõeveer, M., Einasto, J., \& Tago, E. 1978, MNRAS, 185, 357

Kale, R., Dwarakanath, K. S., Lal, D. V., et al. 2016, JApA, 37, 31

Komatsu, E., Dunkley, J., Nolta, M. R., et al. 2009, ApJS, 180, 330

Liddle, A. (ed.) 2003, An Introduction to Modern Cosmology (2nd ed; Chichester: Wiley-VCH), 188

Lietzen, H., Tempel, E., Liivamägi, L. J., et al. 2016, A\&A, 588, L4

Liivamägi, L. J., Tempel, E., \& Saar, E. 2012, A\&A, 539, A80

Martínez, V. J., \& Saar, E. 2002, Statistics of the Galaxy Distribution (Boca Raton, FL: Chapman Hall/CRC)

Nadathur, S. 2016, MNRAS, 461, 358

Nadathur, S., \& Crittenden, R. 2016, ApJL, 830, L19

Nadathur, S., Hotchkiss, S., \& Sarkar, S. 2012, JCAP, 6, 042

Nagamine, K., \& Loeb, A. 2003, NewA, 8, 439

Nishizawa, A. J. 2014, PTEP, 2014, 06B110

Padilla, N. D., Ceccarelli, L., \& Lambas, D. G. 2012, MNRAS, 363, 977

Padmanabhan, T. 1993, Structure Formation in the Universe (Cambridge: Cambridge Univ. Press)

Park, C., Choi, Y.-Y., Kim, J., et al. 2012, ApJL, 759, L7

Pavlidou, V., \& Tomaras, T. N. 2014, JCAP, 9, 020

Peebles, P. J. E. 1980, The Large-Scale Structure of the Universe (Princeton, NJ: Princeton Univ. Press)

Proust, D., Quintana, H., Carrasco, E. R., et al. 2006, A\&A, 447, 133

Raychaudhury, S. 1989, Natur, 342, 251

Reese, E. D., Mroczkowski, T., Menanteau, F., et al. 2012, ApJ, 751, 12

Rines, K., \& Diaferio, A. 2006, AJ, 132, 1275

Rines, K., Geller, M. J., Diaferio, A., \& Kurtz, M. J. 2013, ApJ, 767, 15

Sahni, V., \& Starobinsky, A. 2000, IJMPD, 9, 373

Sarkar, P., Yadav, J., Pandey, B., \& Bharadwaj, S. 2009, MNRAS, 399, L128

Sheth, R. K., \& Diaferio, A. 2011, MNRAS, 417, 2938

Sheth, R. K., Mo, H. J., \& Tormen, G. 2001, MNRAS, 323,

Stoughton, C., Lupton, R. H., Bernardi, M., et al. 2002, AJ, 123, 485

Tully, R. B., Courtois, H., Hoffman, Y., \& Pomarède, D. 2014, Natur, 513, 71

van Weeren, R. J., Röttgering, H. J. A., Bagchi, J., et al. 2009, A\&A, 506, 1083

Vikram, V., Chang, C., Jain, B., et al. 2015, PhRvD, 92, 022006

Wen, Z. L., Han, J. L., \& Liu, F. S. 2012, ApJS, 199, 34 